\title{
Review
}

Jie Huang, Hansi Ma, Dingbo Chen, Huan Yuan, Jinping Zhang, Zikang Li, Jingmin Han, Jiagui Wu* and Junbo Yang*

\section{Digital nanophotonics: the highway to the integration of subwavelength-scale photonics}

\author{
Ultra-compact, multi-function nanophotonic design based on computational inverse design
}

https://doi.org/10.1515/nanoph-2020-0494

Received August 29, 2020; accepted November 23, 2020;

published online December 18, 2020

\begin{abstract}
Nanophotonic devices with high densities are extremely attractive because they can potentially merge photonics and electronics at the nanoscale. However, traditional integrated photonic circuits are designed primarily by manually selecting parameters or employing semi-analytical models. Limited by the small parameter search space, the designed nanophotonic devices generally have a single function, and the footprints reach hundreds of microns. Recently, novel ultra-compact nanophotonic devices with digital structures were proposed. By applying inverse design algorithms, which can search the full parameter space, the proposed devices show extremely compact footprints of a few microns. The results from many groups imply that digital nanophotonics can
\end{abstract}

Jie Huang and Hansi Ma are contributed equally to this Review.

*Corresponding authors: Jiagui $\mathbf{W u}$, College of Electronic and Information Engineering, Southwest University, Chongqing, 400715, China, E-mail: mghswu@gmail.com. https://orcid.org/0000-00032743-5162; and Junbo Yang, Center of Material Science, National University of Defense Technology, Changsha, Hunan, 410073, China; and China State Key Laboratory on Advanced Optical Communication Systems and Networks, Peking University, Beijing, 100871, China, E-mail: yangjunbo@nudt.edu.cn Jie Huang, Hansi Ma and Dingbo Chen, Center of Material Science, National University of Defense Technology, Changsha, Hunan, 410073, China, E-mail: huangjie10@nudt.edu.cn (J. Huang), 201821521328@smail.xtu.edu.cn (H. Ma),

chendingbo15@nudt.edu.cn (D. Chen). https://orcid.org/0000-00023423-5532 (J. Huang)

Huan Yuan, Jinping Zhang and Jingmin Han, College of Electronic and Information Engineering, Southwest University, Chongqing, 400715, China, E-mail: huanyuan1806@email.swu.edu.cn (H. Yuan), zhangjinping@email.swu.edu.cn (J. Zhang), jingminhan@outlook.com (J. Han)

Zikang Li, School of Computer, Electronics and Information, Guangxi University, Nanning, Guangxi, 530004, China,

E-mail: 1721811403@qq.com

Ә Open Access. ( 2020 jie Huang et al., published by De Gruyter. ((c)) BY License. achieve not only ultra-compact single-function devices but also miniaturized multi-function devices and complex functions such as artificial intelligence operations at the nanoscale. Furthermore, to balance the performance and fabrication tolerances of such devices, researchers have developed various solutions, such as adding regularization constraints to digital structures. We believe that with the rapid development of inverse design algorithms and continuous improvements to the nanofabrication process, digital nanophotonics will play a key role in promoting the performance of nanophotonic integration. In this review, we uncover the exciting developments and challenges in this field, analyse and explore potential solutions to these challenges and provide comments on future directions in this field.

Keywords: computational inverse design; digital nanophotonics; fabrication tolerances; ultra-compact nanophotonic devices.

\section{Introduction of ultra-compact nanophotonics and digital nanophotonics}

The field of nanophotonics explores the complex behaviours of light in structures at the subwavelength scale. Due to unremitting scientific exploration and research, the design of ultra-compact nanophotonic devices has attained numerous successes over the past few decades. Moreover, benefiting from the rapid development of high-density nanofabrication technologies, nanophotonic devices have achieved a fairly large scale of practical integration in a number of fields, including optical switches [1, 2], all-optical memories [3, 4], programmable nanophotonic processors [5-7], quantum photonic circuits [8, 9], photonic neural network circuits [10] and other photonic integrated circuits (PICs) [11-14].

Although conventional nanophotonic devices have been widely used in various PICs and even in some 
commercial products [15], due to the lack of general design software such as Very High Speed Integrated Circuit Hardware Description Language (VHDL) and Verilog in the field of electronic integrated circuits (EICs), the components of PICs are designed mostly by manually selecting parameters or by employing semi-analytical models. Hence, limited by the low design efficiency of the traditional design methods and the small parameter search space for conventional nanophotonic devices, the design procedure is often time consuming, and the designed devices generally have large footprints. Considering that a high density corresponds to low power consumption and high performance in the fields of EICs and PICs, we may need to mitigate the low design efficiency of the traditional design methods and avoid the bottleneck caused by the large footprints of single-function devices if we want to integrate tens of billions of components on a single PIC. One potential solution to these problems is to employ inverse-designed digital structures. Specifically, researchers can achieve novel ultra-compact digital nanophotonic devices [16-22] with digital structures [23-26] by utilizing advanced inverse design algorithms [27]. Compared with traditional structures, which originally refer to artificial materials composed of periodic or specially arranged structural elements $[28,29]$, digital structures can provide more degrees of freedom, enabling the propagation of light in nanophotonic devices to be controlled.

As shown in Figure 1, digital nanophotonics primarily involves using inverse design algorithms to design PICs which mainly consist of two materials (such as silicon and silica/air) and could even potentially consist of more materials in the future. It is a sub-field but very active filed of PICs, and the structures employed by digital nanophotonic devices are analogous to the concept of digital metamaterials [30,31]. Digital nanophotonics aids in breaking the limitations of traditional periodic metamaterials and makes the combination of arbitrary topological metamaterials possible, thereby enabling the design of ultracompact single-function devices [16-19, 22, 32], miniaturized multi-function devices [33-35] and even smart devices with super-complex functions [36]. Moreover, by adding regularized constraints to digital structures [32, 33, 37, 38], the performance and fabrication tolerances of these devices can be balanced. The concept of regularized constraints is generalized from the structural optimization problem and is used to filter out the ill-posed solutions of the optimization problem $[39,40]$. In the inverse design of nanophotonic, the regularized constraints usually used to constrain the minimum gap and minimum structure feature size. Researchers have received many good nanophotonic schemes with regularized constraints. For example, Ke $\mathrm{Xu}$ 's group proposed a series of ultra-compact mode-division multiplexed photonic circuits based on regularized digital structures; these circuits can realize an arbitrary signal route with a signal speed of $112 \mathrm{Gbit} / \mathrm{s}$ encoded on each mode [20]. Coincidentally, Jelena Vučković's group reported an on-chip integrated dielectric laser accelerator (DLA) [21] using regularized digital structures enabled by an objective-first algorithm [16]. The energy gain of the presented DLA can reach $0.915 \mathrm{keV}$ within $30 \mu \mathrm{m}$ corresponding to an acceleration gradient of $30.5 \mathrm{MeV} / \mathrm{m}$. With the continuous improvement of nanofabrication technologies and the fusion and normalization of inverse design algorithms, the design of nanophotonic devices will gradually be standardized and modularized with the eventual transition towards large-scale integration.

We intend to uncover the exciting developments and challenges in the field of digital nanophotonics; in doing so, we analyse and explore potential solutions to these challenges and provide comments on future directions in this field. The review is organized as follows: Section 2 summarizes the mainstream optimization design algorithms of nanophotonic devices, including heuristic optimization algorithms, gradient-based algorithms and deep learning. Section 3 provides a review of inverse-designed nanophotonic devices that covers most passive nanophotonic devices and some active devices. Finally, the challenges and opportunities of digital nanophotonics are discussed in Section 4 and the conclusions are presented in Section 5.

\section{Optimization design algorithms of nanophotonic devices}

The demands in the design of ultra-compact nanophotonic devices to establish high-density PICs have sparked the unprecedented development of inverse design algorithms. According to the degree of abstraction, the mainstream optimization algorithms employed for nanophotonic devices can be categorized into three groups: heuristic optimization algorithms, gradient-based algorithms and deep learning. Different algorithms, of course, may involve different simulation methods, such as the finite-difference time-domain (FDTD) method, the finite-difference frequency-domain (FDFD) method, the finite element method (FEM) and rigorous coupled-wave analysis (RCWA), and can also involve different programming languages, such as MATLAB, Python and the scripting languages of commercial products. Although different algorithms, simulation tools and programming languages are intertwined, they are all utilized to find an optimized refractive index 


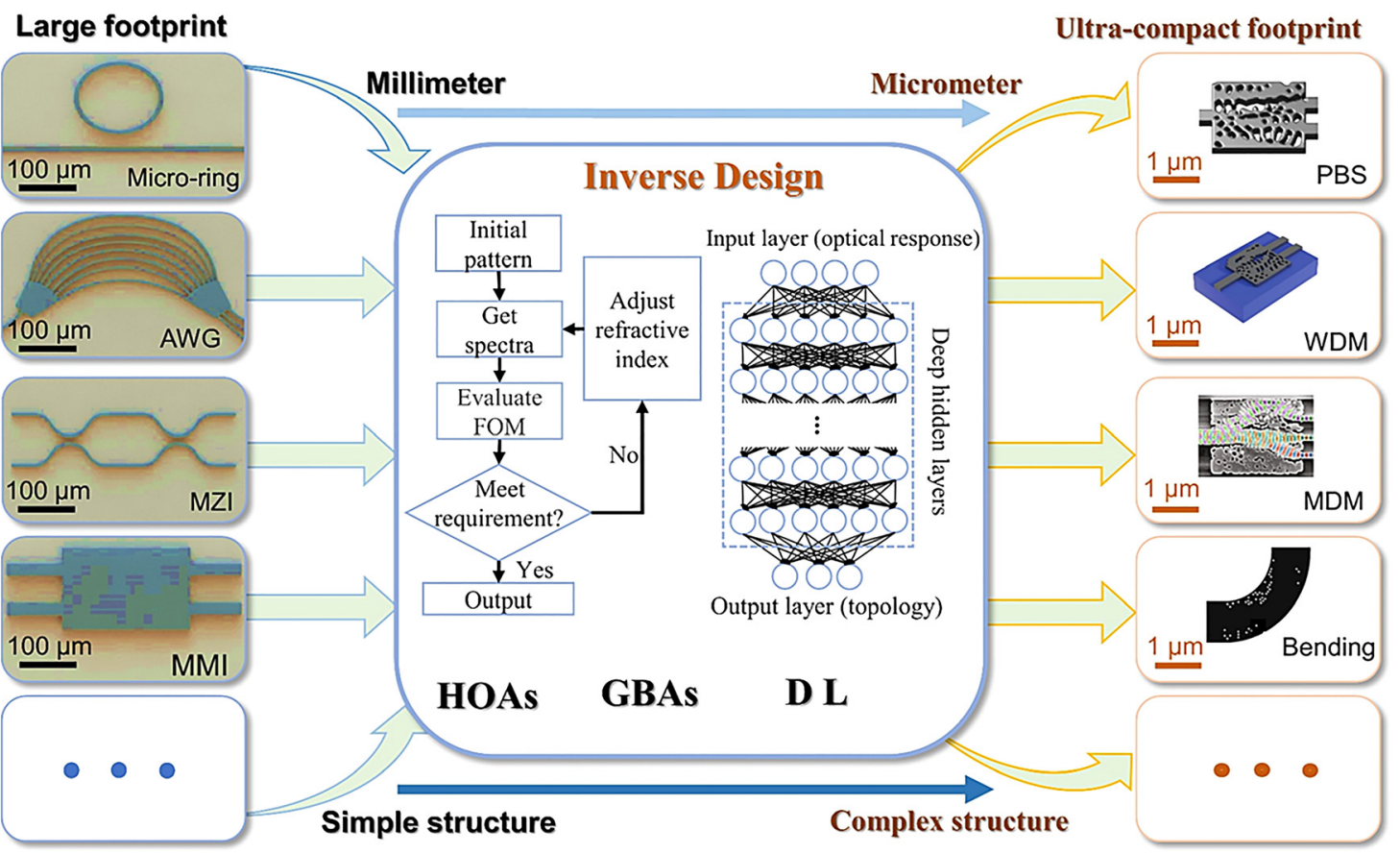

Figure 1: A schematic diagram depicting the methods of computational inverse design and the comparisons between traditional nanophotonic devices and inverse-designed nanophotonic devices.

Left: Traditional devices with large footprints and simple structures, including the Micro-ring, Mach-Zehnder interferometer (MZI), Multimode interference (MMI) coupler and so on. Middle: The optimization processes of computational inverse design and three kinds of associated optimization algorithms, including heuristic optimization algorithms (HOAs), gradient-based algorithms (GBAs) and deep learning (DL). Right: inverse-designed nanophotonic devices with ultra-compact footprints and complex structures, including polarization beam splitter (PBS) (Reproduced with permission [16]. Copyright 2013, Optical Society of America), wavelength-division multiplexing (WDM) (Reproduced with permission [18]. Copyright 2015, Nature Publishing Group), mode-division multiplexing (MDM) (Reproduced with permission [27]. Copyright 2018, Nature Publishing Group), waveguide bending (Reproduced with permission [20]. Copyright 2019, Nature Publishing Group) and so on.

distribution in the design area to fulfil the design requirements. Here, we summarize the optimization algorithms combined with digital structures (discussed in order from simple to complex and from non-gradient to intelligent).

\subsection{Heuristic optimization algorithms}

As one of the most classic algorithms in the optimization field, the genetic algorithm (GA) simulates the process of natural selection and evolution to obtain the optimal solution [41]. The GA was proposed by John Holland in the 1960s [41], and then further developed by him and his colleagues at the University of Michigan [42]. After two decades of development, the GA was widely used in scientific research and engineering. In the field of electromagnetics, the GA is applied mainly in the optimization design of antenna and radar structures [43]. With the emergence of nanophotonic devices intelligently designed with inverse design algorithms in recent years, this computational technique has been introduced into the inverse design of nanophotonic devices [44-46].

In GA-based nanophotonic device design, all individuals with a set of parameters are encoded with special schemes, and the parameters are converted into bit strings called genes. For instance, in the case of the binary encoding discussed here, the values of the parameters (genes) can be either "0" or " 1 ", where " 0 " represents an etched pixel and " 1 " represents an unetched pixel. As schematically demonstrated in Figure 2A, the optimization process of the GA can be divided into three steps: (1) generating the initial populations and calculating the fitness; (2) performing crossover and mutation according to established rules and then regenerating new populations and (3) repeating the fitness function calculation and evolution process until the termination condition is met. In the phase of generating the initial populations, a population where the individuals possess randomly generated genes is formed. Then, simulation tools are employed to evaluate the fitness function corresponding to the userdefined figure of merit (FOM) of the design problem. The 
process of regenerating new populations makes full use of the GA operators: during crossover, the two parent populations are intermixed to generate two children populations, where the crossover point is randomly picked; then, the mutation operator randomly selects some of the genes within the populations and inverts them, after which the current generation of parents is replaced with the new generation of children. As shown in Figure 2B, based on the proposed flow chart in Figure 2A, Zejie Yu et al. proposed on-chip wideband Fabry-Perot (FP) cavities [44]. The initial generation here is composed of 200 randomly generated one-dimensional arrays, where each array represents the etched or unetched pixel distribution of a device. In the optimization process, the authors first used a roulette wheel selection method to select 200 populations from the current generation, and then the crossover and mutation operators were employed to generate a new generation. The optimization process converged after 10
A

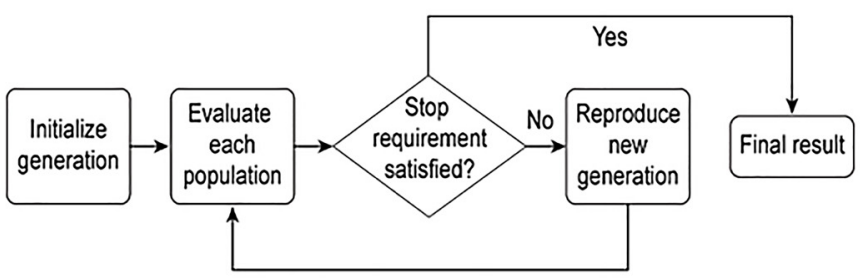

B

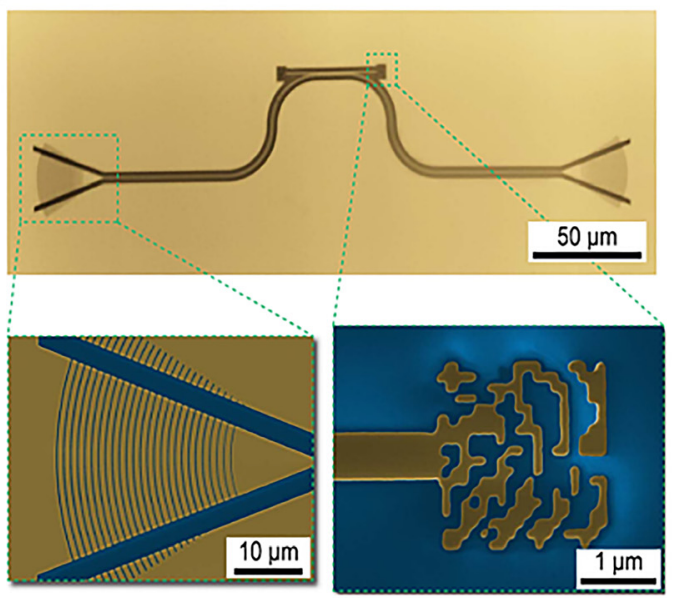

C

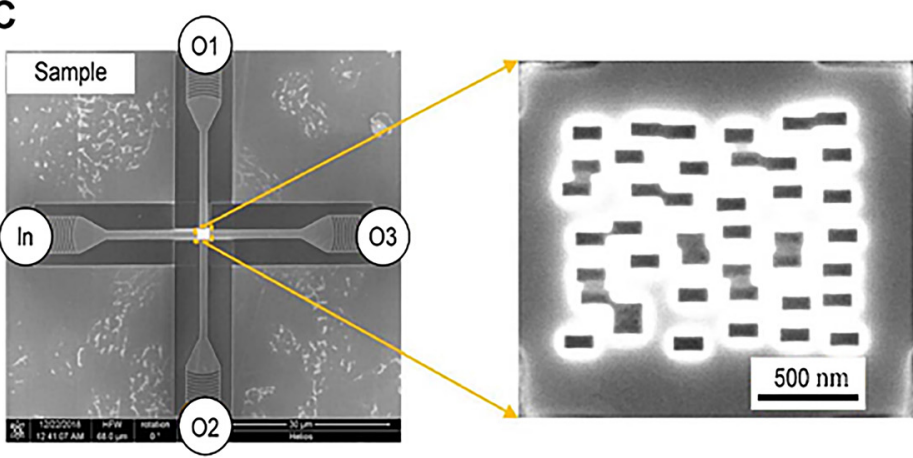

Parent population 1 Crossover point

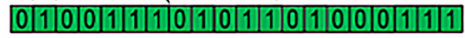

Parent population \& Crossover point $\downarrow_{\text {Crossover }}$

$11111010|1| 0|0| 1|0| 0|1| 0|1| 1|0| 011011$

Children population 1

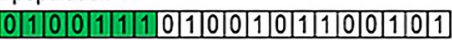

Children population 2

$111110101101011|0| 1|1| 0|1| 0|0| 0|1| 1 \mid 1$
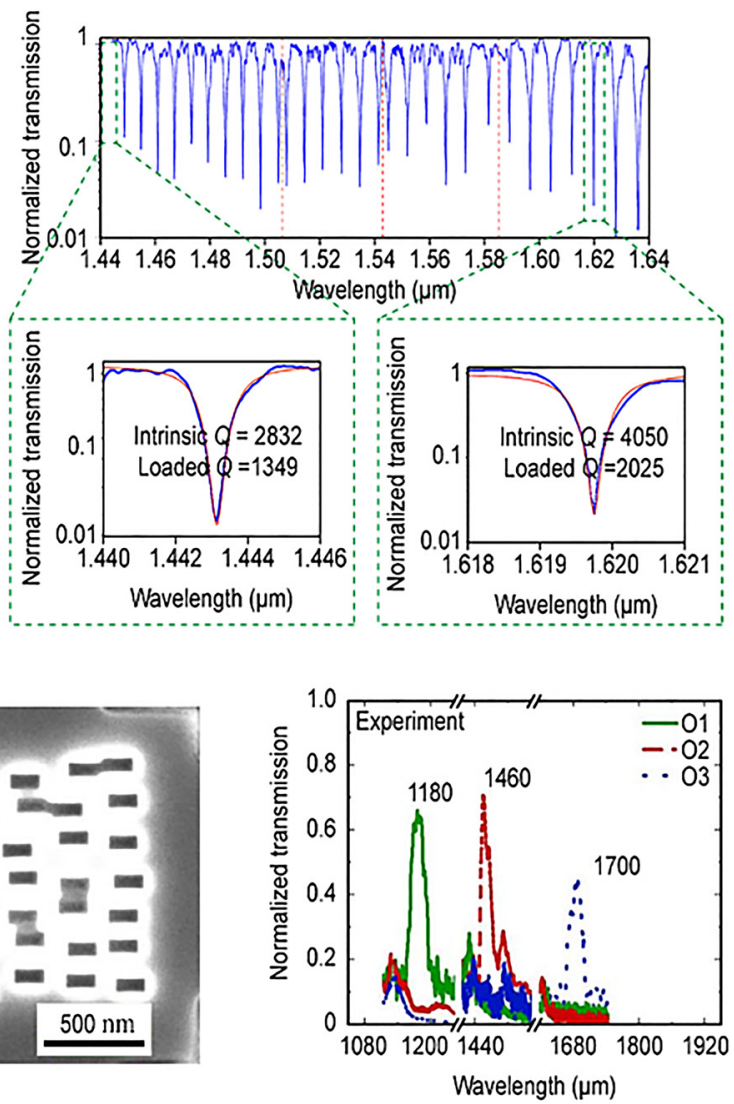

Figure 2: Illustration of the GA.

(A) The flow chart of the optimization process. (B) On-chip wideband FP cavity. Left: optical microscope image of the FP cavity device and magnified scanning electron microscopy (SEM) image of the left grating coupler and the right reflector. Right: Normalized transmission spectrum of the FP cavity device and magnified spectra showing the optical resonances at 1443 and $1620 \mathrm{~nm}$. Reproduced with permission [44]. Copyright 2017, Optical Society of America. (C) Three-channel wavelength router with a footprint of $1.8 \times 1.4 \mu \mathrm{m}$. Left: SEM images of the fabricated device and a magnified SEM image of the centre part of the device. Right: measured transmission spectrum. Reproduced with permission [47]. Copyright 2019, Optical Society of America. 
generations of evolution, and the experimental results indicated that the FP cavities exhibited intrinsic quality factors exceeding 2000 in a spectral width of $200 \mathrm{~nm}$.

We note that to guarantee the fabricability of the designed FP cavities, Yu et al. [44] set the size of the pixels as $120 \times 120 \mathrm{~nm}$. For a digital-structure-based device, the larger the number of pixels is in the design region, the greater the probability of achieving high performance can be. However, for the GA, an increase in the number of optimization variables means that both the number of individuals in the initial population and the number of evolution generations must also increase. If the pixel size of the FP cavities is set to $20 \times 20 \mathrm{~nm}$, the optimization time for the proposed FP cavities may increase from approximately $48 \mathrm{~h}$ to hundreds of hours. In addition, a feature size of $20 \mathrm{~nm}$ is difficult for state-of-the-art fabrication technologies. To extend the design freedom without affecting the fabricability of devices, Zhouhui Liu et al. proposed an intelligent algorithm based on the GA [47]. As demonstrated in Figure $2 \mathrm{C}$ and Table 1 of the study by Liu et al. [47], the design freedom is improved without changing the fabricability of devices by giving some basic geometrical cells with a finite number of different sizes and allowing changes in the cell distribution during the optimization process. The measured experimental results of the designed three-channel wavelength router showed that the proposed scheme keeps the fabricability of the designed devices by setting the proper fabrication size of pixels, and extend the design freedom by featuring pixels of different sizes and non-uniform distributions.

Another heuristic optimization algorithm is the direct binary search (DBS) algorithm, which was first introduced by Michael A. Seldowitz et al. in 1987 [48]. Using this algorithm, Bing Shen and colleagues from the University of Utah reported continuous progress in the design of nanophotonic devices $[49,50]$. As demonstrated in Figure 3A, the device under consideration is first discretized into hundreds of basic pillars called pixels. Similar to the GA, the initial state of the pixels is either randomly generated or specifically designed. Subsequently, a randomly chosen or specifically designed pixel is first perturbed to switch its state, and then the FOM reflecting the performance of the device is calculated. If the FOM increases compared to the initial FOM, the performance of the device is improved, and the state change of this pixel should be maintained; otherwise, the performance is not improved, and the pixel returns to the previous state [49]. An iteration ends when all the pixels are toggled. Figure 3B shows the smallest polarization beam splitter (PBS) demonstrated at that time, which was designed using the DBS algorithm [50]. The experimental results showed that the average transmission efficiency of the PBS was greater than $70 \%$ and that the extinction ratio was greater than $10 \mathrm{~dB}$ within a bandwidth of $32 \mathrm{~nm}$.

Due to the distinct point scanning mode of the DBS algorithm, the optimization process is highly likely to prematurely converge to a locally optimal solution. Although this issue can be mitigated by parallelizing the optimization process with different starting points [50], this approach will greatly increase the time and economic cost and can become particularly problematic when the optimization objective function becomes more complex or the number of pixels in the optimization area increases. To accelerate the convergence speed, Xiang Wen et al. proposed dynamically adjusting the convergence radius of the objective function [51]. In addition to the design efficiency, the fabrication tolerances of the designed devices constitute another research topic in the inverse design of nanophotonics that has yet to be resolved. Accordingly, Luluzi Lu et al. proposed a scheme of replacing square holes with round holes for etching, which can effectively reduce the fabrication error caused by incomplete etching at the corners of square holes [52].

In recent years, the DBS algorithm has received extensive attention from researchers due to its simplicity and strong scalability, and a series of high-performance devices have been demonstrated using the original DBS algorithm $[20,53,54]$ or modified DBS algorithm $[55,56]$. For example, Figure 3C shows the design results of modedivision multiplexing (MDM) circuits whose components were designed by the DBS algorithm [20]. The experimental results show that the routing of high-speed signals was achieved. However, limited by state-of-the-art fabrication technologies, to guarantee the fabricability of the designed devices, all these schemes still place strong regularized constraints on the devices. Consequently, to further increase the performance of nanophotonic devices, Hansi Ma et al. proposed the rotatable direct binary search (RDBS) algorithm [35]. In the RDBS algorithm, the authors did not rigidly add regularized constraints to the parameters of pixels. Rather, they flexibly relaxed the regularization conditions to allow other parameters of the pixels, such as the shape and rotation angle, to be changeable. As a modified DBS algorithm, the RDBS algorithm still evaluates the pixels individually; the difference is that the RDBS algorithm searches for other parameter spaces, such as the shape and rotation angle. By employing the RDBS algorithm, the authors flexibly and selectively optimized the suitable pixel parameters that greatly affected the performance of devices and finally achieved a device with high performance and high fabrication tolerances. For instance, they designed an ultra-compact $1 \times 2$ mode 
A

C

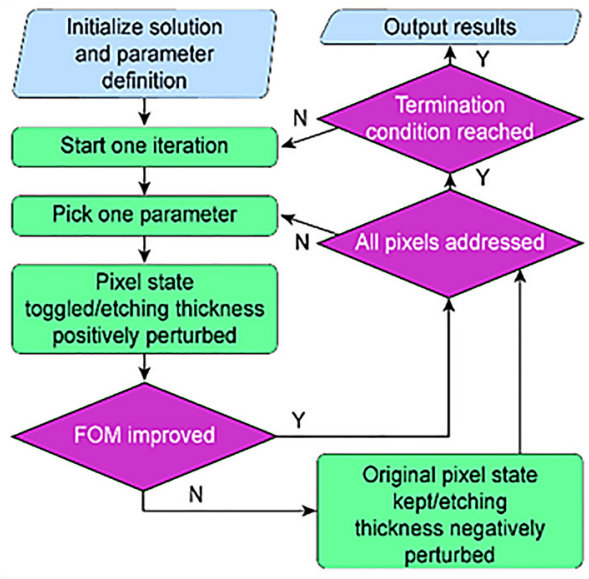

B
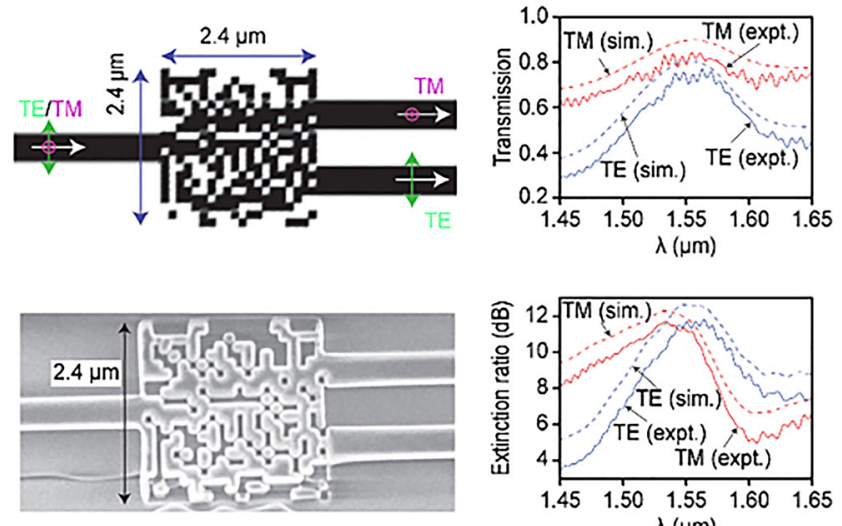
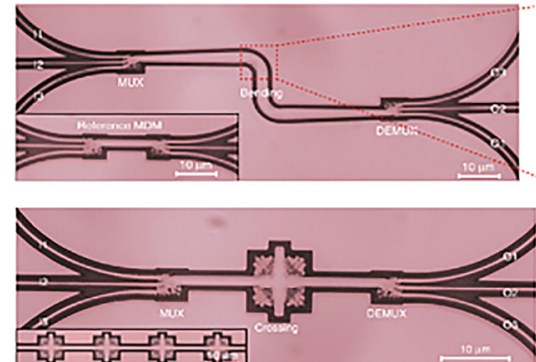
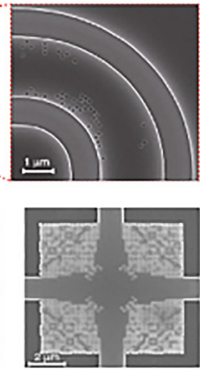
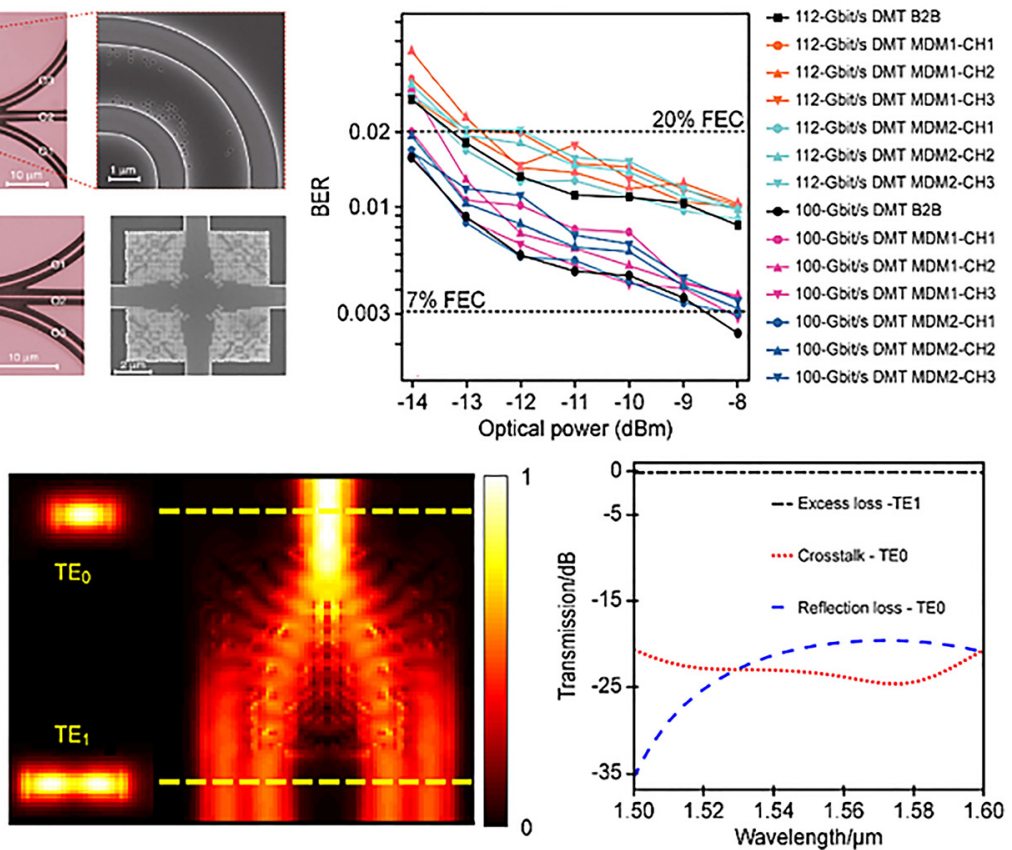

Figure 3: Illustration of the DBS algorithm.

(A) Flow chart of the DBS algorithm. Reproduced with permission [49]. Copyright 2015, Optical Society of America. (B) Integratednanophotonics PBS. Upper left: geometry of the PBS. Lower left: SEM image of the fabricated PBS. Upper right: measured and simulated transmission efficiencies of the PBS. Lower right: measured and simulated extinction ratios of the PBS. Reproduced with permission [50]. Copyright 2015, Nature Publishing Group. (C) Mode-division multiplexed photonic circuits that can be arbitrarily routed. Upper left: SEM image of the mode-division multiplexing (MDM) circuit consisting of a multiplexer (MUX), bending and demultiplexer (DEMUX). Lower left: SEM image of the MDM circuit consisting of MUX, crossing and DEMUX. Right: measured bit error rate curves for the back-to-back and MDM transmissions of 100 and $112 \mathrm{Gbit} / \mathrm{s}$ discrete multi-tones. Reproduced with permission [20]. Copyright 2019, Nature Publishing Group. (D) Ultra-compact $1 \times 2$ mode converter. Left: Geometry of the converter. Middle: simulated steady-state light intensity distribution at a wavelength of $1550 \mathrm{~nm}$. Right: Simulated transmission efficiency, extinction ratio and reflection of the converter. Reproduced with permission [35]. Copyright 2020, Optical Society of America.

converter using the RDBS algorithm that can simultaneously realize power splitting and mode conversion (see Figure 3D).

In addition to the GA and the DBS algorithm, other heuristic optimization algorithms, such as the particle swarm optimization (PSO) algorithm [57-59] and simulated annealing algorithm [60,61], are also widely used in the design of nanophotonic devices. As such, heuristic optimization algorithms have made great strides in designing nanophotonic devices based on digital structures with strong regularization constraints [20, 35, $44-47,49-56,58,61]$, periodic metamaterials $[62,63]$ and 
metamaterials guided by analytical models [64]. However, the low design efficiencies and strong regularization constraints of heuristic optimization algorithms can make it difficult to balance the performances and fabrication tolerances of complex devices.

\subsection{Gradient-based algorithms}

As mentioned before, a series of high-performance nanophotonic devices have been designed by employing heuristic optimization algorithms. However, the computation cost exponentially increases if the device size increases because heuristic optimization algorithms adopt a hard search method that ignores the gradient information of the FOM function. Although the time cost can be effectively reduced by parallel computing, the economic cost will increase. Therefore, optimization algorithms based on the gradient information of the FOM function have arisen and become another potential solution to the inverse design problems of nanophotonic devices.

The gradient-based optimization algorithm provides a mathematical abstraction of the inverse design problems of nanophotonic devices. First, the optimization parameters and the mapping relationship between the optimization parameters and the permittivity distribution of the design region are specified. Then, the derivative of the FOM function with respect to the optimization parameters is obtained. To ensure that the derivative is not limited by the number of optimization parameters, the adjoint analysis technique has been widely used [65]. Lastly, with the derivative of the FOM function, the optimized permittivity distribution of the design region can be achieved by applying methods such as steepest descent $[66,67]$. According to the complexity of the mathematical model, the gradient-based optimization algorithms can be divided into three categories, namely, the shape optimization algorithm [68-70], the topology optimization (TO) algorithm $[71,72]$ and the objective-first (OB-1) algorithm [16, 73].

The shape optimization algorithm, also known as the boundary optimization algorithm, achieves the design target by optimizing the shapes of the boundaries between different materials. Because the shape optimization algorithm changes the contour structure only within the design region, the optimized devices are much easier to fabricate than those optimized by heuristic optimization algorithms. Without considering the size of the device, the shape optimization algorithm is especially suitable for device designs that have no strict requirements on size but have strict requirements on the loss and extinction ratio. For example, as shown in Figure 4A, Andrew Michaels and
Eli Yablonovitch designed a non-adiabatic waveguide taper between a narrow and a wide waveguide [69]. The insertion loss of the designed taper was only $0.041 \mathrm{~dB}$ at $1550 \mathrm{~nm}$. However, although this non-adiabatic waveguide taper has great advantages in loss compared to a digital metamaterial-based taper designed by the DBS algorithm [74], the taper remains disadvantaged by its large footprint. In other words, the shape optimization algorithm is not suitable for designing ultra-compact devices and multifunction devices.

To reduce the large size and avoid the single-function nature of the devices designed by the shape optimization algorithm, Nicolas Lebbe and colleagues proposed the multi-level shape optimization algorithm [70]. As shown in Figure 4B, they successfully designed a novel photonic polarization rotator with a polarization conversion efficiency of approximately $98.5 \%$, an insertion loss of less than $0.35 \mathrm{~dB}$ within a bandwidth of $100 \mathrm{~nm}$, and, most importantly, a footprint of $6 \times 1 \mu \mathrm{m}$. We found that the design degree of freedom is increased due to the application of the multi-level shape optimization scheme. However, these shape optimization algorithms involve a stronger regularization constraint than that of the abovementioned heuristic optimization algorithms; as a result, the shape optimization algorithms cannot control light at the subwavelength scale as effectively as digital structures designed by the heuristic optimization algorithms.

The TO algorithm was first presented by Martin Philip Bendsøe and Noboru Kikuchi in 1988 [71]. In contrast to the shape optimization algorithms, the TO algorithm allows permittivity in the design region to adopt arbitrary topological geometries. Because the size of the discrete pixels in the design region can be infinitely reduced, TO-based devices can control light at the subwavelength scale. In addition, the TO algorithm considers the gradient information of the FOM function and exhibits stronger "directivity" during optimization; thus, its convergence speed is generally much faster than that of the heuristic optimization algorithms. In addition, during practical optimization, the computational cost of the TO process and the fabricability of the optimized devices must be fully considered, which means that reliable regularization constraint schemes must be employed [75, 76]. Researchers from the Technical University of Denmark have successfully achieved a series of planar photonic crystal (PhC)-based novel nanophotonic devices [77], including bent waveguides [78, 79], $Y$-splitters [80] and mode converters [81]. As demonstrated in Figure $4 \mathrm{C}$, the designed $\mathrm{PhC}$ structures can achieve a previously unattainable ultra-compact device size, new functionality and high performance using the constrained TO algorithm. For 
A

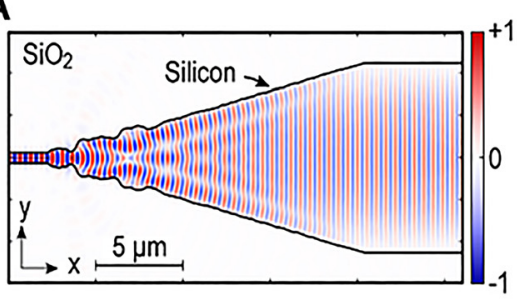

C

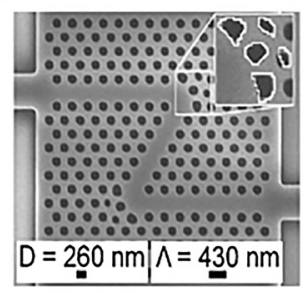

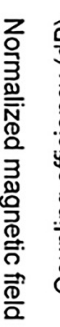

B

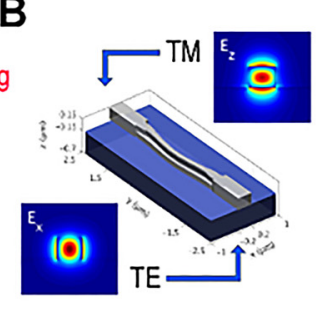

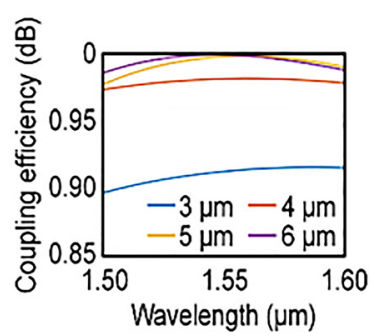
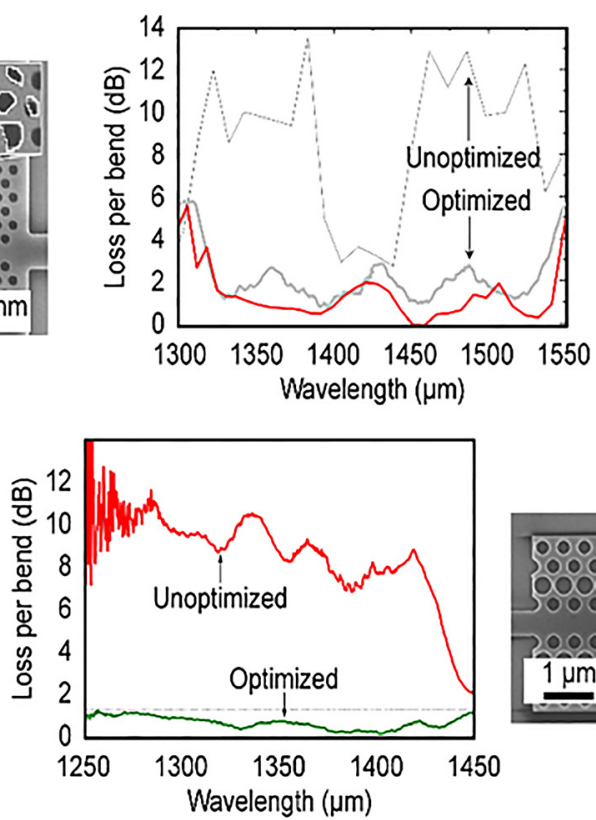
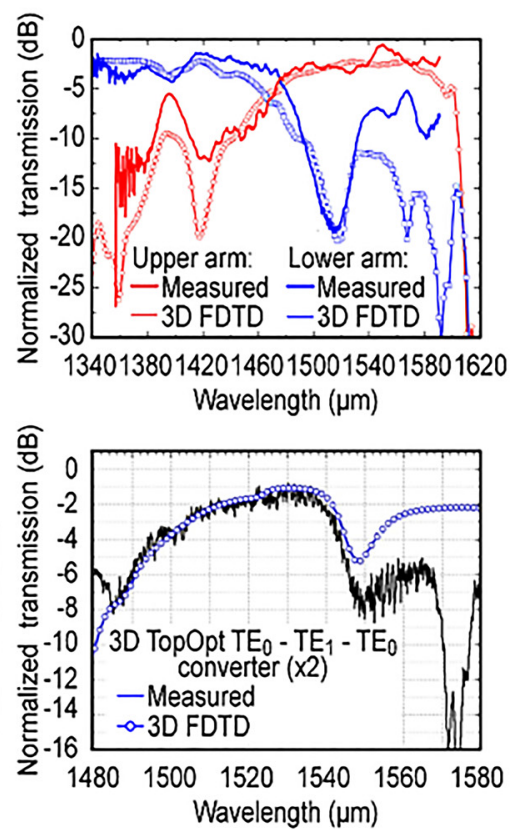

Figure 4: Illustration of the shape optimization and TO algorithms.

(A) Non-adiabatic waveguide taper. Left: optimized structure overlaid with the real part of the frequency domain. Right: coupling efficiency of the designed taper. Reproduced with permission [69]. Copyright 2018, Optical Society of America. (B) Left: Schematic of a two-level photonic polarization rotator. Right: the polarization conversion efficiency of the rotator for different lengths. Reproduced with permission [70]. Copyright 2019, Optical Society of America. (C) Upper left: SEM image of the designed Z-bend waveguide and the simulated and measured loss per bend under TE-polarized light. Reproduced with permission [78]. Copyright 2004, Optical Society of America. Lower left: SEM image of the topology-optimized bends and measured loss per bend for the unoptimized $60^{\circ}$ bends (red) and the topology-optimized $60^{\circ}$ bends (green). Reproduced with permission [79]. Copyright 2004, Optical Society of America. Upper right: SEM image of the fabricated $Y$-splitter and the 3D FDTD-calculated and measured transmission. Reproduced with permission [80]. Copyright 2007, Optical Society of America. Lower right: SEM image of the fabricated mode converter and the measured and simulated transmission spectra for the X 2 mode converter. Reproduced with permission [81]. Copyright 2014, Optical Society of America.

example, by applying a $Z$-bend waveguide consisting of two successive $120^{\circ} \mathrm{PhC}$ waveguide bends, exceptional transmission can be experimentally obtained where the transverse electric (TE)-polarized light shows extremely low loss in a large bandwidth of more than $200 \mathrm{~nm}$ [78]. However, all the above-mentioned devices are based on partly optimized $\mathrm{PhC}$ structures; that is, only some specific regions of the $\mathrm{PhC}$ structure have been designed by the constrained TO algorithm. Similar to the shape optimization algorithm, the limited parameter space can cause a problem with the device size; for instance, the size of the mode converter based on the TO-algorithm-optimized PhC structure [81] is larger than that designed by a heuristic optimization algorithm [46]. Consequently, the above researchers developed the improved TO algorithm, which can search the full parameter space of the design region. A low-loss and broadband two-mode (de)multiplexer has been reported, and the experimental results indicate that the designed (de)multiplexer can realize high-performance (de)multiplexing with a footprint of $2.6 \times 4.22 \mu \mathrm{m}$ [82]. In addition to researchers from different research groups, engineers from commercial software companies [83] and opensource software organizations [84] have actively promoted shape optimization and TO research in recent years. 
Nanophotonic device design problems involving Maxwell's equations are not convex optimization problems. Hence, when local optimization algorithms such as the steepest descent method are used, the final optimization result is inevitably related to the initial structure of the design region. If a proper initial structure is not selected, the final optimization result may converge undesirably. Unfortunately, the initial structure of the design region obtained by using the above-mentioned shape optimization and TO algorithms is generally selected at random. Although a randomly selected initial structure can converge to a locally optimal solution in most cases, the optimization processes are likely to not converge or to converge to unacceptable results when the size of the device has strict restrictions and the functionality is relatively complex. In 2012, Jesse Lu proposed the concept of the OB-1 algorithm $[16,73]$. Unlike the shape optimization algorithm or TO algorithm, the OB-1 algorithm first forces the design objectives to be satisfied but allows Maxwell's equations to be violated (the "physics residual"); then, by using the alternating direction method of multipliers (ADMM) algorithm [85], the physics residual is minimized. The OB-1 algorithm gives priority to the optimization goal during the optimization process; thus, although OB-1 is not a global optimization algorithm, its optimization results are often close to the target performance. However, the permittivity distribution of the design region is allowed to change continuously within the set range during the OB-1 optimization process, which means that the designed devices cannot be fabricated; nevertheless, the permittivity distribution can be used as a starting point for a local optimization algorithm. Thus, combining the initial structure with regularization constraints [32, 33, 86], fabricable nanophotonic devices with ultra-compact footprints and high performance can be achieved using local optimization algorithms.

As one of the best algorithms in the inverse design of nanophotonic devices, the OB-1 algorithm yields fast convergence. Over the past few years, the design of nanophotonic devices with the OB-1 algorithm has experienced rapid development. By using the OB-1 algorithm and subsequently applying local optimization algorithms, a series of key components of PICs have been designed (see Figure 5), including wavelength splitters [18, 87-89], onchip integrated laser-driven particle accelerators [21], focussing wavelength demultiplexers [33], non-reciprocal pulse routers [90], power splitters [91], optical diodes [92], PBSs [93] and other important nanophotonic devices.

\subsection{Deep learning}

Deep learning is a sub-field of machine learning that uses a multitude of nonlinear information processing and abstraction methods for supervised and unsupervised feature learning as well as presentation, classification and pattern recognition [94, 95]. Since David E. Rumelhart et al. first introduced the concept of deep learning to machine learning in 1986 [96] and proposed the back-propagation algorithm, after decades of development, deep learning has been extensively applied in computer vision [97-99], natural language processing [100], speech recognition [101, 102], strategy development [103] and other fields [104-106]. In recent years, well-trained deep neural networks (DNNs) have become able to almost instantaneously find solutions to inverse design problems of nanophotonic devices from an enormous parameter space; accordingly, the deep learning method has gradually been used by researchers to design nanophotonic devices [27, 107-126]. In contrast to ordinary optimization algorithms, once the forward prediction (inverse design) mode has been trained successfully, the deep learning method can predict (inverse design) the spectral response (topological structure) of nanophotonic devices almost instantaneously [116].

The core idea of applying deep learning to nanophotonic device design is to effectively model the relationship between the structural parameters of a device and the optical response as a bidirectional mapping. The problem is that completely different structural parameters may produce the same optical response, and an inconsistent design parameter-optical response mapping will create conflicting instances in the dataset, which will make it difficult for the neural network to converge. To solve this problem, Dianjing Liu et al. proposed the tandem network scheme [109]. By using this scheme, the authors designed a series of $\mathrm{SiO}_{2}$ and $\mathrm{Si}_{3} \mathrm{~N}_{4}$ thin films with Gaussian shape transmission spectra. The design results show that the target response and modelled response almost perfectly match. Because deep learning is a data-driven method, the size and effectiveness of the training dataset strongly determine the quality of the resulting inverse design model [110, 111]. However, the training dataset is usually obtained through full-wave electromagnetic simulations, which require considerable amounts of time and economic costs. To mitigate this issue, researchers have investigated the special optical device design problem in which analytical models can be used to calculate spectral responses. For example, John Peurifoy et al. successfully used artificial neural networks to solve the multilayer nanoparticle-based light scattering problem [112]. In addition to the above-mentioned devices, deep learning methods have achieved remarkable success in designing other types of nanophotonic devices, including plasmonic metamaterials [113-115], chiral metamaterials [116], plasmonic waveguides [117] and topological photonic devices $[118,119]$. Several recent review articles that systematically 
A
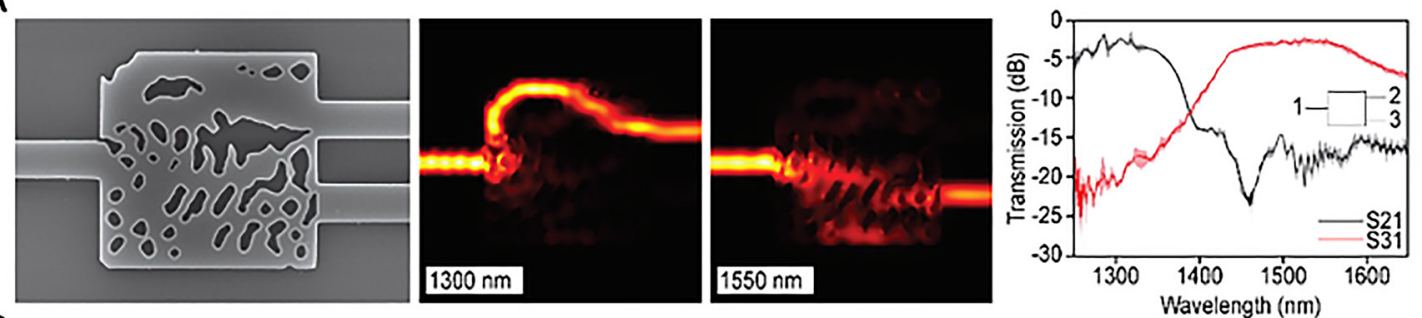

B
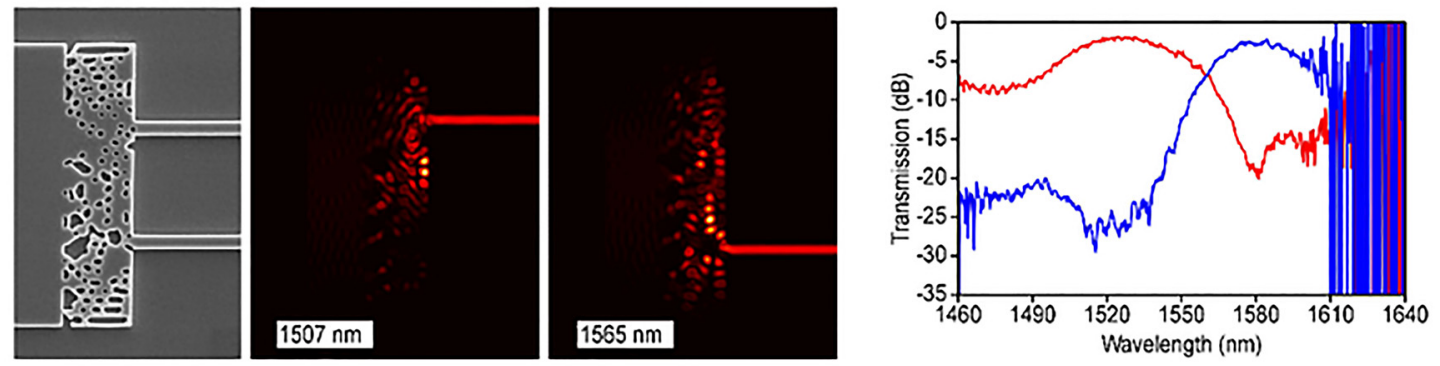

C
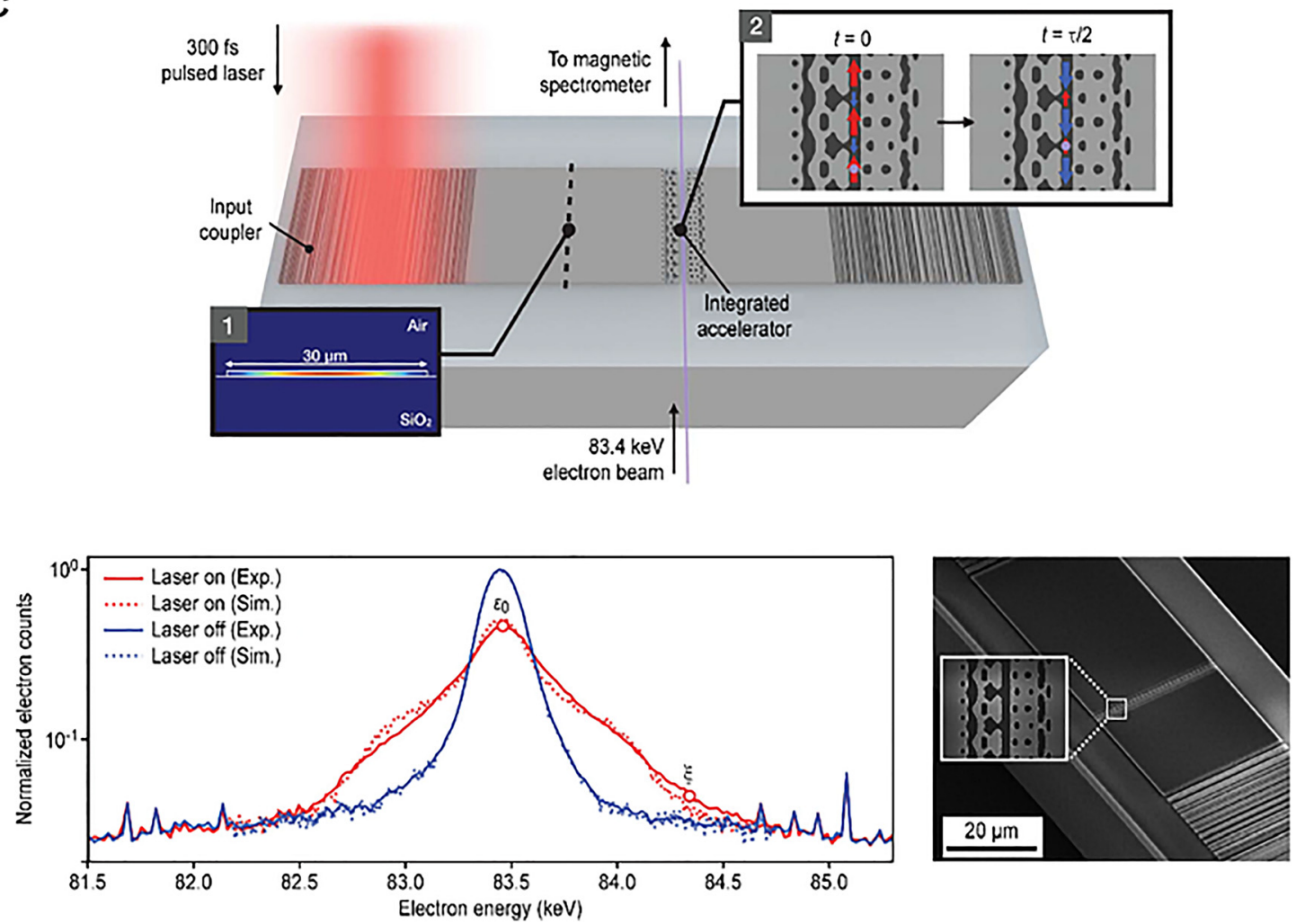

Figure 5: Part of a nanophotonic device designed by the OB-1 algorithm.

(A) Broadband on-chip wavelength demultiplexer. Left: SEM image of the fabricated wavelength demultiplexer. Middle: simulated electromagnetic energy density in a horizontal slice through the middle of the device. Right: Measured $S$-parameters for the device, where $S_{i j}$ is the transmission from port $j$ to port $i$. Reproduced with permission [18]. Copyright 2015, Nature Publishing Group. (B) Two-channel focussing wavelength demultiplexer. Left: SEM image of the fabricated focussing wavelength demultiplexer. Middle: Simulated electromagnetic energy density in a horizontal slice through the middle of the device. Right: Measured $S$-parameters for the device, where $S_{i j}$ is the transmission from port $j$ to port $i$. Reproduced with permission [33]. Copyright 2020, De Gruyter. (C) On-chip integrated laser-driven particle accelerator. Upper left: Schematic depicting the components of the on-chip accelerator composed of pairs of inverse-designed grating couplers and the inversedesigned integrated accelerator. Lower left: Electron energy spectrum (log scale) without an incident laser (blue curve) and with an incident laser ( $3.0 \mathrm{~mW}, 335 \mathrm{MV} / \mathrm{m}$ peak field, $\lambda=1.94 \mu \mathrm{m}$; red curve) on the grating coupler. Right: SEM image of a single-stage accelerator at 30 periods fabricated on a 500-nm silicon on insulator (SOI) stack. Reproduced with permission [21]. Copyright 2020, American Association for the Advancement of Science. 
summarized the advances obtained by applying deep learning to the design of nanophotonic devices are referred to the reader $[25,107,108]$.

It is worth noting that most of the above-mentioned inverse design problems involve only a limited number of design parameters. When deep-learning-assisted inverse design is applied to a situation where thousands of design parameters are present $[18,50]$, the deep learning models can be difficult to train with a limited number of sample points from the design space. Although dimensionality reduction techniques [108] such as principal component analysis [121], the Fourier transform [122] and autoencoders [123] can effectively reduce the design parameters in the design space, the application scope of these techniques is limited. Therefore, the design of digitalstructure-based nanophotonic devices, as demonstrated in the studies by Pigott et al. [18] and Shen et al. [50], remains an unsolved problem. Interestingly, by combining the DBS algorithm with a deep learning method, Mohammad $\mathrm{H}$. Tahersima et al. designed a series of $1 \times 2$ power splitters with a training dataset that contains only approximately 20,000 simulation data points [124]. Although this model has limited generalization ability, it provides a potential solution for using a deep-learning-enabled design method to design digital-structure-based nanophotonic devices.

\section{Trends of inverse-designed nanophotonic devices}

Digital nanophotonics, which is concomitant with inversedesigned digital structures, is rapidly becoming popular in the field of nanophotonic integration, and the past few years have witnessed its gradual transition from singlefunction devices [16-19, 50] to PICs [20, 21]. At the same time, software engineers have been actively promoting related inverse design methods for large-scale applications $[83,84]$. Digital-structure-based devices have been shown to be compatible with industry-standard photonic foundries [38]. Consequently, with the rapid development of inverse design algorithms, many digital-structure-based devices have been designed, including most passive nanophotonic devices and some active devices. Reviewing the history of EICs, we find that digital nanophotonics is as important to PICs as digital circuits are to EICs. This section focuses on the evolutionary trends of inverse-designed nanophotonic devices, including the trends from singlemode devices to multi-mode devices, from single-function devices to multi-function devices and smart devices with super-complex functions, and from simulation results to the design of highly robust devices.

\subsection{The trend from single-mode devices to multi-mode devices}

For simplicity, simple (single-mode) devices are typically designed first, including waveguide crossing, which support only the fundamental TE mode [127, 128], singlemode waveguide bends [129], two-channel wavelength demultiplexers $[18,130]$, two-channel mode (de)multiplexers [80] and two-channel power splitters [52]. However, to further enhance the transmission and processing of information as in high-density PICs, the devices must support more than one mode. For example, a waveguide crossing is an indispensable component of a PIC, as illustrated in Figure 6A. To realize the high-performance crossing of light, Pablo Sanchis and colleagues demonstrated a GA-optimized crossing structure based on a mode expander [127]. The designed device exhibits transmission losses lower than $0.2 \mathrm{~dB}$, and the crosstalk and reflection losses are lower than $-40 \mathrm{~dB}$ within a bandwidth of $20 \mathrm{~nm}$. Coincidentally, by using the PSO algorithm, Yi Zhang et al. designed a complementary metal-oxide-semiconductor (CMOS)compatible single-mode waveguide crossing with an average insertion loss of $0.18 \pm 0.03 \mathrm{~dB}$ and a crosstalk of $-41 \pm 2 \mathrm{~dB}$ [128]. Many efforts have been made to achieve multi-mode crossing; dual-mode [131] and three-mode [20] waveguide crossings were realized in succession (see Figure $6 \mathrm{~B}$ and Figure $6 \mathrm{C}$ ). With the combination of the DBS algorithm and digital structures, in which the discrete units are optimized instead of the shapes of the boundaries, the above-mentioned devices can support two and three modes with compact footprints of only $4.8 \times 4.8 \mu \mathrm{m}$ and $8 \times 8 \mu \mathrm{m}$, respectively. For the dual-mode crossing, the measured insertion losses and crosstalk are less than $0.6 \mathrm{~dB}$ and $-24 \mathrm{~dB}$, respectively, from 1530 to $1590 \mathrm{~nm}$ for both the $T E_{0}$ mode and the $T E_{1}$ mode [131]. Similarly, for the threemode waveguide crossing, measurements show that the insertion losses are $0.28,0.68$ and $0.82 \mathrm{~dB}$ for the $T E_{0}, T E_{1}$ and $T E_{2}$ modes, respectively, and the crosstalk is less than $-20 \mathrm{~dB}$ for all three modes [20].

Another important component of a PIC is the waveguide bend. Although the modes of guided waves can be well confined in straight waveguides, the optical field can be distorted and can suffer from large radiation loss in curved planar waveguides, especially in sharp-corner waveguides. By introducing a natural spline section, W. Bogaerts and S. K. Selvaraja dramatically reduced the bending loss of the bend waveguides. For instance, the use of a $10^{\circ}$ spline section can reduce the bend loss of a bend waveguide from 0.037 to $0.009 \mathrm{~dB}$ with $3 \mu \mathrm{m}$ bend radii [132]. However, although the introduction of spline sections induces a small footprint penalty, this approach is difficult to extend to the 
A
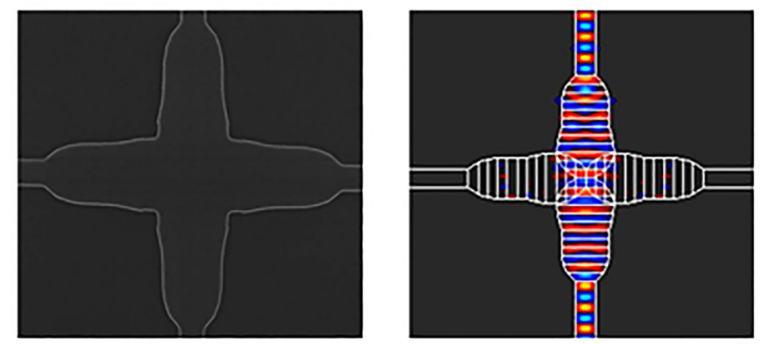

C
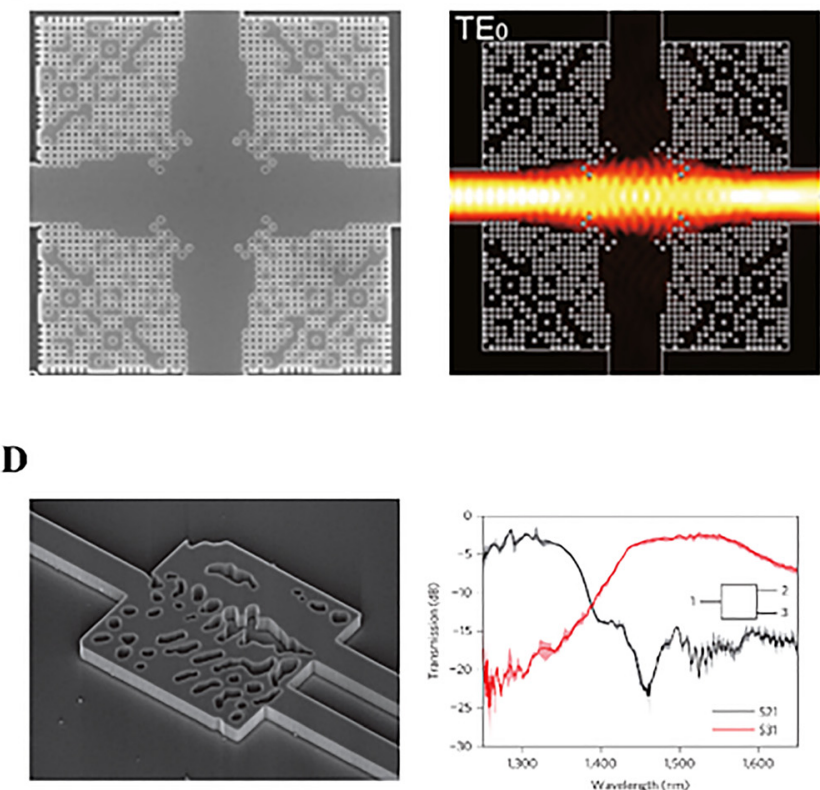

$\mathbf{F}$

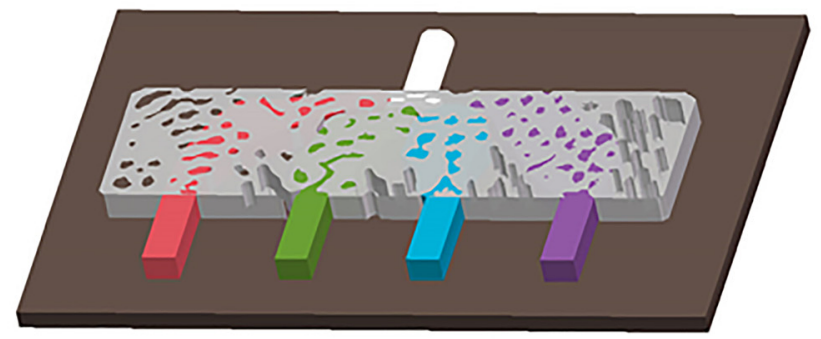

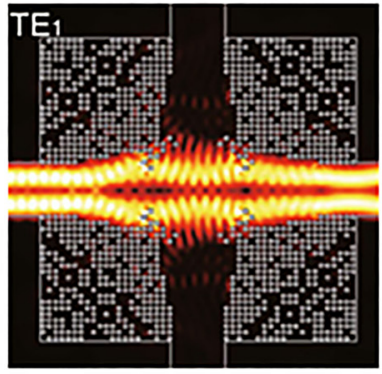

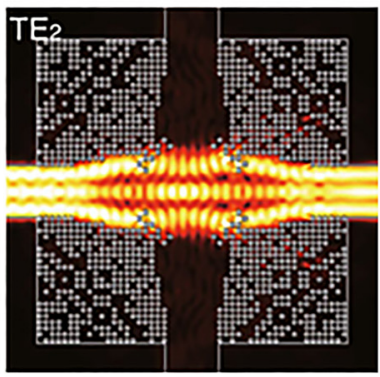

$\mathbf{E}$

$\mathbf{B}$
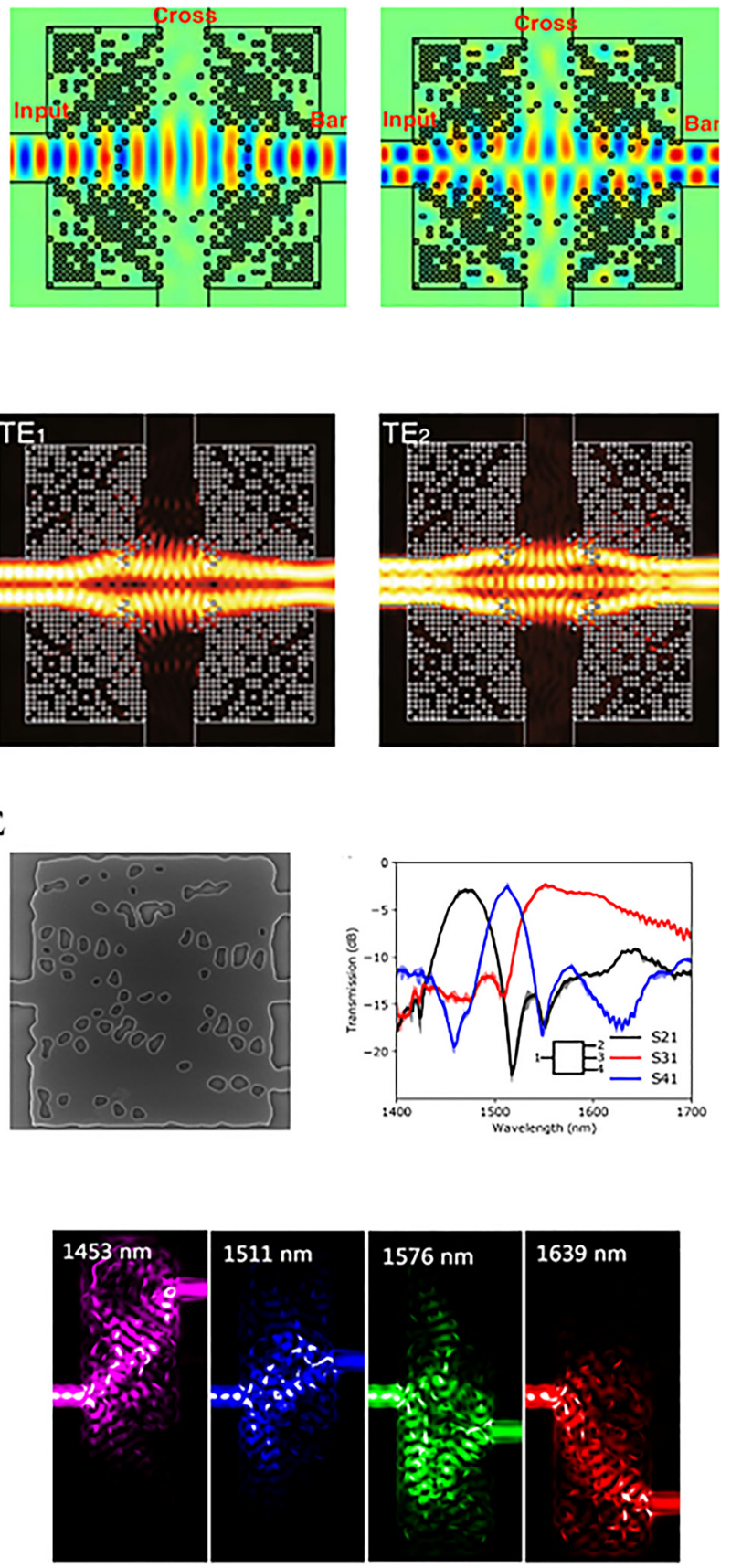

Figure 6: Diagram of the development of inverse-designed devices from single-mode (two-channel) to multi-mode (multi-channel) devices. (A) Inverse-designed single-mode waveguide crossing. Left: SEM image of the fabricated waveguide crossing structure. Right: electric field distribution of the device. Reproduced with permission [127]. Copyright 2009, Optical Society of America. (B) Inverse-designed dual-mode waveguide crossing. Left: Simulated magnetic field distributions of $H_{z}$ for the $T E_{0}$ mode. Right: Simulated magnetic field distributions of $H_{z}$ for the $T E_{1}$ Mode. Reproduced with permission [131]. Copyright 2018, Optical Society of America. (C) Inverse-designed three-mode waveguide crossing. Left: SEM image of the fabricated crossing. Right: Simulated optical field distributions for the $T E_{0}, T E_{1}$ and $T E_{2}$ modes. Reproduced with permission [20]. Copyright 2019, Nature Publishing Group. (D) Inverse-designed two-channel wavelength demultiplexer. Left: SEM image of the fabricated demultiplexer. Right: Measured $S$-parameters for three identical demultiplexers. Reproduced with permission [18]. Copyright 2015, Nature Publishing Group. (E) Inverse-designed three-channel wavelength demultiplexer. Left: SEM image of the fabricated demultiplexer. Right: Measured S-parameters for four identical devices. Reproduced with permission [87]. Copyright 2017, American Chemical Society. (F) Inverse-designed tuneable four-channel wavelength demultiplexer. Left: Three-dimensional diagram of the designed demultiplexer. Right: Simulated electromagnetic energy distribution for different wavelengths. Reproduced with permission [89]. Copyright 2020, Elsevier B.V. 
design of multi-mode bent waveguides due to the limited number of tuneable parameters in the design process. By using the DBS algorithm, which can search a far larger parameter space than other algorithms, YingJie Liu et al. described a set of sharply bent waveguides with varying widths and radii. The fabricated waveguides, with a width of $2 \mu \mathrm{m}$ and a bending radius of $1 \mu \mathrm{m}$, show an incredibly low insertion loss $(1 \mathrm{~dB})$ and an extremely high suppression ratio (>20 dB) to higher-order modes [133]. Although the insertion loss of the proposed bent waveguides based on digital structures is relatively large, the footprints of the devices are much smaller than those proposed in the study by Bogaerts and Selvaraja [132]. To support higher-order modes, Chunlei Sun et al. proposed and experimentally demonstrated a sharply bent two-mode silicon waveguide by introducing a pair of mode converters [134]. By engineering the geometry of the mode converters with the PSO algorithm, the mode mismatch between multi-mode straight and bent waveguides can be effectively avoided. The measured results indicated that the insertion loss is lower than $0.2 \mathrm{~dB}$ and that the crosstalk is less than $-22 \mathrm{~dB}$ with a bending radius of $5 \mu \mathrm{m}$ from 1500 to $1600 \mathrm{~nm}$. In addition, YingJie Liu et al. homoplastically designed a three-mode bent waveguide [20], for which the measured loss is less than 0.71, 0.74 and $0.95 \mathrm{~dB}$ for the $T E_{0}, T E_{1}$ and $T E_{2}$ modes, respectively, and the measured crosstalk is lower than $-20 \mathrm{~dB}$ for all three modes from 1500 to $1580 \mathrm{~nm}$.

As key components of PICs, digital-structure-based wavelength demultiplexers have also been designed with inverse design methods. As illustrated in Figure 6D, researchers have designed a two-channel wavelength demultiplexer; the measured insertion loss is approximately $2 \mathrm{~dB}$, and the crosstalk is lower than $-11 \mathrm{~dB}$ [18]. Since a wavelength (de)multiplexer can multiply the data processing capacity of a PIC by the number of wavelength channels used [135-137], the information processing capability increases with the number of channels. As a result, the multi-channel wavelength demultiplexers were designed [87-89]. As shown in Figure 6E, Logan Su et al. experimentally achieved a three-channel wavelength demultiplexer with a $40 \mathrm{~nm}$ spacing (1500, 1540 and $1580 \mathrm{~nm}$ ) and a footprint of $24.75 \mu^{2}$ [87]. The measured peak insertion loss of the fabricated demultiplexer is $-2.29 \mathrm{~dB}$, and the crosstalk is lower than $-10.7 \mathrm{~dB}$. After that, Jingmin Han et al. designed a tuneable four-channel wavelength demultiplexer [89]. The simulated peak insertion loss of the demultiplexer is greater than $-2.10 \mathrm{~dB}$, and the crosstalk is lower than $-13.2 \mathrm{~dB}$ (see Figure 6F).

In addition to wavelength-division multiplexing (WDM), the mode-division multiplexing (MDM) technique is important for further improving the information processing capability of PICs [138-140]. Therefore, the design of a high-performance mode (de)multiplexer with a compact footprint, low insertion and low crosstalk is an essential task for realizing a densely integrated MDM system [141]. For example, Louise F. Frellsen et al. designed a topology-optimized mode (de)multiplexer that (de)multiplexes the $T E_{0}$ and $T E_{1}$ modes in a silicon photonic wire waveguide [82]. The experimental results indicated that the insertion loss is less than $1.2 \mathrm{~dB}$ within a bandwidth of $100 \mathrm{~nm}$, the measured crosstalk is lower than $-12 \mathrm{~dB}$, and the extinction ratio exceeds $14 \mathrm{~dB}$ in the C-band. Subsequently, researchers demonstrated three-mode [20, 142] and four-mode (de)multiplexers [143] using the DBS algorithm. For example, Hucheng Xie et al. proposed and experimentally demonstrated an ultra-compact mode multiplexer for the $T E_{0}, T E_{1}, T E_{2}$ and $T E_{3}$ modes [143]. The measured loss of this multiplexer is less than $1.5 \mathrm{~dB}$, and the inter-mode crosstalk is approximately $-20 \mathrm{~dB}$ at $1550 \mathrm{~nm}$ and lower than $-14.6 \mathrm{~dB}$ within $1510-1570 \mathrm{~nm}$.

In addition to the above-mentioned waveguide crossings, bent waveguides, wavelength multiplexers and mode (de)multiplexers, other components of PICs, such as power splitters, have also experienced similar development processes, i.e. from two-channel devices to multi-channel devices [52, 124, 144-150]. Based on Figure 6, we can clearly conclude that inverse-designed devices have rapidly developed from supporting single-mode to supporting multi-mode devices. In fact, if the concept of the "mode" is further extended, the gradual development trend of inverse-designed devices from a single wavelength to multiple wavelengths $[18,50,93,151]$ can also be attributed to the increase in the mode. For example, by employing the OB-1 algorithm, Jie Huang et al. extended the operational bandwidth of an inverse-designed PBS from a single wavelength [50] to multiple wavelengths [93]. The simulated insertion losses are less than $0.4 \mathrm{~dB}$ $(0.35 \mathrm{~dB})$ for the TE (transverse magnetic, TM) mode at wavelengths of 1310,1550 and $1600 \mathrm{~nm}$, and the extinction ratios of both polarization states are more than $14.5 \mathrm{~dB}$ in the C-band, L-band and O-band.

\subsection{The trend towards multi- functionalization and robust design}

A device generally has only one function, such as crossing, bending, wavelength demultiplexing or mode de(multiplexing). However, in some specific application scenarios or PICs with particularly high integration requirements, it may be necessary for a device to have multiple functions simultaneously. Examples include 
simultaneous fibre-to-chip coupling and wavelength demultiplexing [152], focussing and wavelength demultiplexing [33], mode converting and polarization splitting [50], or mode converting and power splitting [35]. As shown in Figure 7A and B, researchers have designed two kinds of multi-function devices based on the OB-1 algorithm and DBS algorithm. The first is a wavelength demultiplexing grating coupler that can couple verticalincident broadband Gaussian beams to separate waveguides [152]. The measured splitting ratios of the two output ports are $17 \pm 2 \mathrm{~dB}$ at $1310 \mathrm{~nm}$ (O-band) and $12 \pm 2 \mathrm{~dB}$ at $1540 \mathrm{~nm}$ (C-band). The second kind of device is a modeconverting PBS that can realize mode compression and splitting with a footprint of $4 \times 3 \mu \mathrm{m}$. The measured peak transmission efficiency for the TE (TM) mode is $58 \%$ (71\%), and the corresponding extinction ratio is $13.88 \mathrm{~dB}$
(13.77 dB). In addition to the simple superposition of functions, inverse-designed devices can also implement complex functionalities that are nearly intelligent. For example, Nasim Mohammadi Estakhri et al. introduced inverse-designed metastructures that can solve integral equations by using monochromatic electromagnetic fields [36]. As depicted in Figure 7C, the designed metastructures were experimentally shown to solve a generic integral equation at microwave frequencies. The measured results show good agreement with the expected theoretical results and the full-wave simulations.

In contrast to the traditional design approach, which tunes a few geometric parameters, the introduced inverse design methods have made great achievements in designing nanophotonic devices by capitalizing on the increased design degrees of freedom. However, to
A

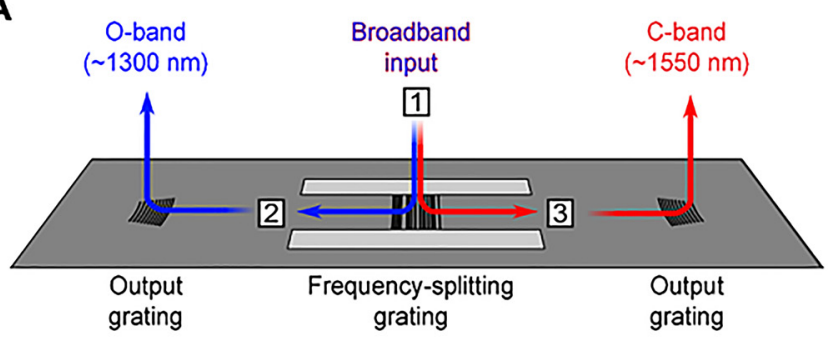

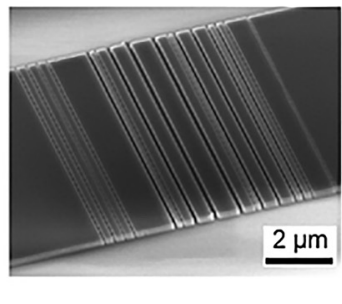

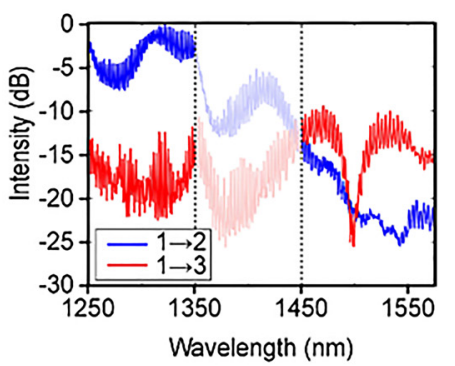

B
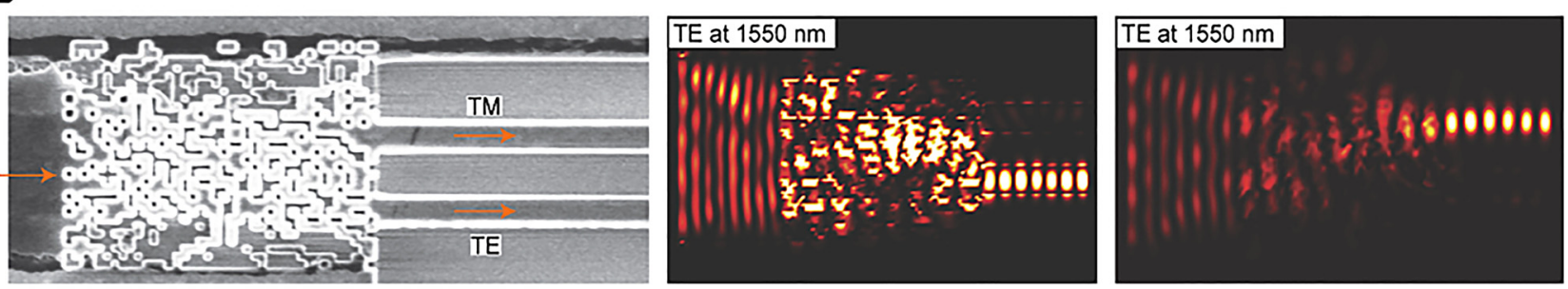

C
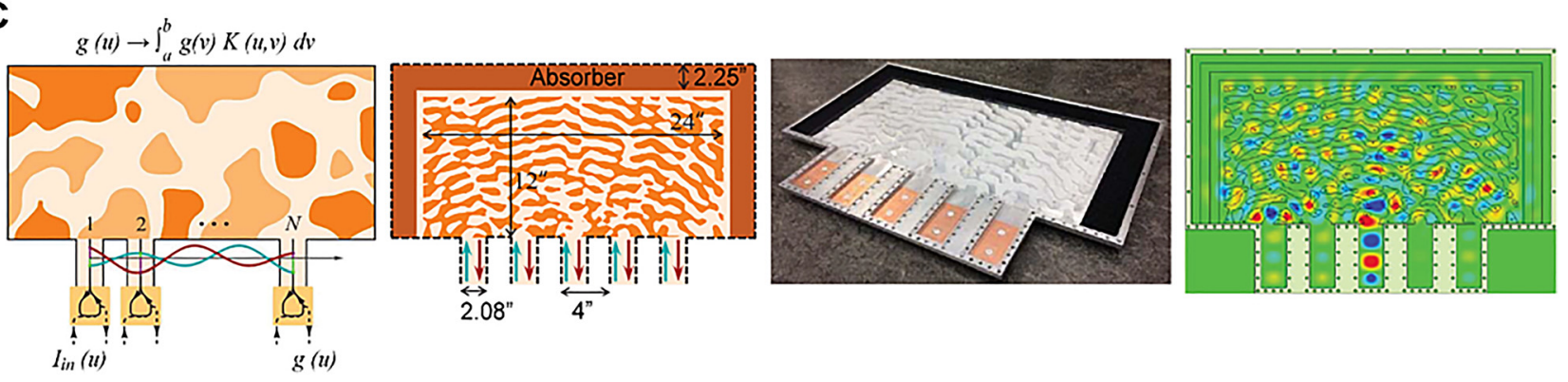

Figure 7: Inverse-designed multi-function devices.

(A) Wavelength demultiplexing grating coupler. Left: schematic diagram of the designed WDM grating. Middle: SEM image of the fabricated grating. Right: Measured coupling efficiencies of the device. Reproduced with permission [152]. Copyright 2014, Nature Publishing Group. (B) Mode-converting PBS. Left: SEM image of the fabricated mode-converting PBS. Middle and Right: Simulated intensity distributions of the TE and TM modes at a wavelength of $1550 \mathrm{~nm}$. Reproduced with permission [50]. Copyright 2015, Nature Publishing Group. (C) Left: Conceptual sketch of the integral solver, which is composed of an inverse-designed metamaterial kernel, $N$ in/out coupling elements and $N$ connecting waveguides. Left-middle: The inverse-designed distribution of the relative permittivity of the kernel. Right-middle: photograph of the constructed metamaterial kernel. Right: simulated stable electric field distribution of the solver when light is excited at port 3. Reproduced with permission [36]. Copyright 2019, American Association for the Advancement of Science. 
guarantee the fabricability of the designed devices, further fabrication constraints may need to be added during the inverse design process $[32,33,35,37,38,44,47,50,52,86]$. Specifically, a robust design is usually achieved by adding regularization constraints, including constraints on the minimum gap and minimum structure feature size. For example, in the optimization process of heuristic optimization algorithms, the size of discrete square pixels in the design region of a device is generally larger than or equal to $100 \times 100 \mathrm{~nm}[44,47,50]$ to facilitate fabrication. To further reduce the impact of fabrication imperfections, Luluzi $\mathrm{Lu}$ et al. proposed the replacement of square air holes with round air holes for etching [52], which can effectively reduce the fabrication errors caused by the reactive ion etching (RIE) lag effect [153]. When gradient-based algorithms are employed in the inverse design process, a number of regularization constraint schemes are available, including the addition of more regularization terms to the structural parameters [32, 86], the use of different types of density filters [33, 37, 75-77, 154], the employment of morphology-based methods [155] and the use of $b$-spline surfaces [156]. Compared to heuristic optimization algorithms, where all of the pixels in the design region are binarized, the gradient-based algorithms usually generate "grey" areas where the permittivity varies continuously. Thus, binarization schemes such as biasing [22, 87], projection $[33,37,76,157]$ and sigmoid functions [86] are needed.

\section{Challenges and opportunities}

After more than 10 years of development, nanophotonic devices based on inverse-designed digital structures have been extensively applied in most fields of nanophotonics, including passive devices [18, 50], active devices [37, 158-160], metasurfaces [64, 114, 161-165], plasmonics and graphene [117, 166-168] and quantum optics [169-171]. However, several important issues have yet to be mitigated.

First, the designed devices, which are composed of digital structures, generally exhibit strong scattering, which is usually accompanied by energy loss and crosstalk. This makes it difficult to design nanophotonic devices with remarkably high extinction ratios or incredibly low insertion losses. One possible solution is to develop better regularization constraint schemes that consider both the spectral response of the target port and the electromagnetic field distribution in the design region. Through the application of improved regularization constraint schemes, the designed devices can minimize scattering as much as possible while ensuring fabricability.

Second, all the above-mentioned optimization algorithms require repeated calls to the Maxwell equation solver during the optimization process. Hence, although the computing speed can be improved to some extent by parallel computing, the optimization of nanophotonic devices requires considerable time and economic costs, and this problem is especially prominent for the heuristic optimization algorithms. All of the simulation tools (FDTD, FDFD, FEM and RCWA) for Maxwell's equations rely on central processing units (CPUs) and graphics processing units (GPUs) or other common processing chips. Alternatively, we believe one of the potential solutions to this issue is to use artificial intelligence (AI)-accelerated dedicated processors or all-optical AI processors [10].

Third, once an optimization model has been established, the optimal design of nanophotonic devices is subsequently abstracted into a mathematical problem, which means we know very little about the physics of how an optimized structure can achieve a particular function. In addition, since the optimal design of nanophotonic devices is generally not a convex optimization problem, the final optimization results are often related to the initial structure; thus, if we do not understand the physical mechanism, it is difficult to choose the appropriate initial conditions, optimization algorithm and regularization conditions. Although some discussion has occurred in this context regarding about the physical mechanism [53], computational bounds [172] and initial conditions [86, 173], these problems have not been fundamentally and systematically resolved. Addressing these problems will enable the optimal design of nanophotonic devices to become more efficient, and the performances of the designed devices will improve.

Finally, although digital nanophotonics has developed rapidly and achieved remarkable successes in many fields, most of the designed devices based on digital structures are currently devices with simple functions. Consequently, the efficient design of multi-function and high-performance devices is still an urgent problem to be solved. In addition, due to the diversity of the available algorithms, devices with the same function may have completely different topologies and fabrication techniques. Therefore, moving towards the large-scale integration of digital nanophotonics, in addition to the fusion and normalization of different types of optimization algorithms, it will be indispensable to standardize the design process for different types of nanophotonic devices, to form a process design kit (PDK), and to eventually create a common design tool similar to VHDL and Verilog in the EIC field. 


\section{Conclusions}

In this review, we have summarized the recent progress in digital nanophotonics enabled by inverse-designed digital structures. By employing advanced inverse design algorithms such as heuristic optimization algorithms, gradient-based algorithms and deep learning, the designed nanophotonic devices can achieve previously unattainable functionalities with an ultra-compact footprint. For example, by engineering the refractive index distribution of the design region, the designed devices can perform multiple functions simultaneously. More importantly, with nanofabrication technology, which has gradually matured in recent years, the field of digital nanophotonics is gradually developing into a highly integrated technology. Although some problems remain in the design of devices with complex functions and/or super-large footprints and devices with a high extinction ratio or an ultra-low insertion loss, we expect this new field to solve the problem of designing large-scale PICs.

Acknowledgements: We thank Dr. Yongzhuang Zhou for his helpful discussion.

Author contributions: All the authors have accepted responsibility for the entire content of this submitted manuscript and approved submission.

Research funding: This work was supported by the National Natural Science Foundation of China (60907003, 61805278, 61875168), the China Postdoctoral Science Foundation (2018M633704, 2017M612885), the Foundation of NUDT (JC13-02-13, ZK17-03-01), the Hunan Provincial Natural Science Foundation of China (13JJ3001), a Chongqing Postdoctoral Science Foundation Special Funded Project (Xm2017008), and the Fundamental Research Funds for the Central Universities (XDJK2019B059).

Conflict of interest statement: The authors declare no conflicts of interest regarding this article.

\section{References}

[1] T. J. Seok, N. Quack, S. Han, R. S. Muller, and M. C. Wu, "Large-scale broadband digital silicon photonic switches with vertical adiabatic couplers," Optica, vol. 3, pp. 64-70, 2016.

[2] T. J Seok, K. Kwon, J. Henriksson, J. Luo, and M. C. Wu, "Wafer-scale silicon photonic switches beyond die size limit," Optica, vol. 6, pp. 490-494, 2019.

[3] K. Nozaki, A. Shinya, S. Matsuo, et al., "Ultralow-power all-optical RAM based on nanocavities," Nat. Photonics, vol. 6, pp. 248-252, 2012.
[4] E. Kuramochi, K. Nozaki, A. Shinya, et al., "Large-scale integration of wavelength-addressable all-optical memories on a photonic crystal chip," Nat. Photonics, vol. 8, pp. 474-481, 2014.

[5] N. C. Harris, G. R. Steinbrecher, M. Prabhu, et al., "Quantum transport simulations in a programmable nanophotonic processor," Nat. Photonics, vol. 11, pp. 447-452, 2017.

[6] D. Pérez, G. Ivana, and J. Capmany, "Programmable multifunctional integrated nanophotonics," Nanophotonics, vol. 7, pp. 1351-1371, 2018.

[7] N. C. Harris, J. Carolan, D. Bunandar, et al., "Linear programmable nanophotonic processors,” Optica, vol. 5, pp. 1623-1631, 2018.

[8] N. C. Harris, D. Bunandar, M. Pant, et al., "Large-scale quantum photonic circuits in silicon," Nanophotonics, vol. 5, pp. 456-468, 2016.

[9] J. Wang, S. Paesani, Y. Ding, et al., "Multidimensional quantum entanglement with large-scale integrated optics," Science, vol. 360, pp. 285-291, 2018.

[10] Y. Shen, N. C. Harris, S. Skirlo, et al., "Deep learning with coherent nanophotonic circuits," Nat. Photonics, vol. 11, pp. 441-446, 2017.

[11] J. Sun, E. Timurdogan, A. Yaacobi, E. S. Hosseini, and M. R. Watts, "Large-scale nanophotonic phased array," Nature, vol. 493, pp. 195-199, 2013.

[12] Z. Wang, T. Li, A. Soman, D. Mao, T. Kananen, and T. Gu, “On-chip wavefront shaping with dielectric metasurface," Nat. Commun., vol. 10, p. 3547, 2019.

[13] Y. H. Lai, M. G. Suh, Y. K. Lu, et al., "Earth rotation measured by a chip-scale ring laser gyroscope," Nat. Photonics, vol. 14, pp. 345-349, 2020.

[14] J. Wu, S. W. Huang, Y. Huang, et al., "Mesoscopic chaos mediated by Drude electron-hole plasma in silicon optomechanical oscillators," Nat. Commun., vol. 8, p. 15570, 2017.

[15] https://ark.intel.com/content/www/us/en/ark/products/ 188986/intel-silicon-photonics-100g-cwdm4-qsfp28-extendedtemperature-optical-transceiver.html.

[16] J. Lu and J. Vuckovic, "Nanophotonic computational design," Opt. Express, vol. 21, pp. 13351-13367, 2013.

[17] B. Shen, P. Wang, R. Polson, and R. Menon, "Ultra-highefficiency metamaterial polarizer," Optica, vol. 1, pp. 356-360, 2014.

[18] A. Y. Piggott, J. Lu, K. G. Lagoudakis, J. Petykiewicz, T. M. Babinec, and J. Vuckovic, "Inverse design and demonstration of a compact and broadband on-chip wavelength demultiplexer," Nat. Photonics, vol. 9, pp. 374-377, 2015.

[19] B. Shen, R. Polson, and R. Menon, "Integrated digital metamaterials enables ultra-compact optical diodes," Opt. Express, vol. 23, pp. 10847-10855, 2015.

[20] Y. Liu, K. Xu, S. Wang, et al., "Arbitrarily routed mode-division multiplexed photonic circuits for dense integration," Nat. Commun., vol. 10, p. 3263, 2019.

[21] N. V. Sapra, K. Y. Yang, D. Vercruysse, et al., “On-chip integrated laser-driven particle accelerator," Science, vol. 367, pp. 79-83, 2020.

[22] K. Wang, X. Ren, W. Chang, L. Lu, D. Liu, and M. Zhang, “Inverse design of digital nanophotonic devices using the adjoint method," Photonics Res., vol. 8, pp. 528-533, 2020. 
[23] S. Jahani and Z. Jacob, "All-dielectric metamaterials," Nat. Nanotechnol., vol. 11, pp. 23-36, 2016.

[24] I. Staude and J. Schilling, "Metamaterial-inspired silicon nanophotonics," Nat. Photonics, vol. 11, pp. 274-284, 2017.

[25] K. Yao, R. Unni, and Y. Zheng, "Intelligent nanophotonics: merging photonics and artificial intelligence at the nanoscale," Nanophotonics, vol. 8, pp. 339-366, 2019.

[26] S. So, N. Park, H. J. Lee, and J. Rho, "New trends in nanophotonics,” Nanophotonics, vol. 9, pp. 983-985, 2020.

[27] S. Molesky, Z. Lin, A. Y. Piggott, W. Jin, J. Vuckovic, and A. W. Rodriguez, "Inverse design in nanophotonics," Nat. Photonics, vol. 12, pp. 659-670, 2018.

[28] S. Choudhury, D. Wang, K. Chaudhuri, et al., "Material platforms for optical metasurfaces,” Nanophotonics, vol. 7, pp. 959-987, 2018.

[29] P. Cheben, R. Halir, J. H. Schmid, H. A. Atwater, and D. R. Smith, "Subwavelength integrated photonics," Nature, vol. 560, pp. 565-572, 2018.

[30] C. Della Giovampaola and N. Engheta, "Digital metamaterials," Nat. Mater., vol. 13, pp. 1115-1121, 2014.

[31] T. Cui, M. Qi, X. Wan, J. Zhao, and Q. Cheng, "Coding metamaterials, digital metamaterials and programmable metamaterials," Light Sci. Appl., vol. 3, p. e218, 2014.

[32] A. Y. Piggott, J. Petykiewicz, L. Su, and J. Vuckovic, "Fabricationconstrained nanophotonic inverse design," Sci. Rep., vol. 7, p. 1786, 2017.

[33] J. Huang, J. Yang, D. Chen, et al., "Implementation of on-chip multi-channel focusing wavelength demultiplexer with regularized digital metamaterials," Nanophotonics, vol. 9, pp. 159-166, 2019.

[34] H. Ma, J. Huang, K. Zhang, and J. Yang, "Inverse-designed arbitrary-input and ultra-compact $1 \times \mathrm{N}$ power splitters based on high symmetric structure," Sci. Rep., vol.10, p. 11757, 2020.

[35] H. Ma, J. Huang, K. Zhang, and J. Yang, "Ultra-compact and efficient $1 \times 2$ mode converters based on rotatable directbinary-search algorithm," Opt. Express, vol. 28, pp. 17010-17019, 2020.

[36] N. M. Estakhri, B. Edwards, and N. Engheta, “Inverse-designed metastructures that solve equations," Science, vol. 363, pp. 1333-1338, 2019.

[37] T. W. Hughes, M. Minkov, I. A. D. Williamson, and S. Fan, “Adjoint method and inverse design for nonlinear nanophotonic devices," ACS Photonics, vol. 5, pp. 4781-4787, 2018.

[38] A. Y. Piggott, E. Y. Ma, L. Su, et al., "Inverse-designed photonics for semiconductor foundries," ACS Photonics, vol. 7, pp. 569-575, 2020.

[39] C. Keno-Tung and N. Olhoff, "Regularized formulation for optimal design of axisymmetric plates," Int. J. Solid Struct., vol. 18, pp. 153-169, 1982.

[40] M. P. Bendsoe, "Optimal shape design as a material distribution problem,” Struct. Optim., vol. 1, 1989. https://doi.org/10.1007/ BF01650949.

[41] M. Mitchell, An Introduction to Genetic Algorithms, Cambridge, UK, MIT Press, 1998.

[42] J. H. Holland, Adaptation in Natural and Artificial Systems, Ann Arbor, Mich, University of Michigan Press, 1975.

[43] R. L. Haupt and D. H. Werner, Genetic Algorithms in Electromagnetics, Hoboken, NJ, Wiley-IEEE Press, 2007.
[44] Z. Yu, H. Cui, and X. Sun, "Genetically optimized on-chip wideband ultracompact reflectors and Fabry-Perot cavities," Photonics Res., vol. 5, pp. B15-B19, 2017.

[45] T. Feichtner, O. Selig, M. Kiunke, and B. Hecht, "Evolutionary optimization of optical antennas," Phys. Rev. Lett., vol. 109, p. 127701, 2012.

[46] Z. Yu, H. Cui, and X. Sun, "Genetic-algorithm-optimized wideband on-chip polarization rotator with an ultrasmall footprint," Opt. Lett., vol. 42, pp. 3093-3096, 2017.

[47] Z. Liu, X. Liu, Z. Xiao, et al., "Integrated nanophotonic wavelength router based on an intelligent algorithm," Optica, vol. 6, pp. 1367-1373, 2019.

[48] M. A. Seldowitz, J. PAllebach, and D. W. Sweeney, "Synthesis of digital holograms by direct binary search," Appl. Opt., vol. 26, pp. 2788-2789, 1987.

[49] B. Shen, R. Polson, and R. Menon, "Broadband asymmetric light transmission via all-dielectric digital metasurfaces," Opt. Express, vol. 23, pp. 20961-20970, 2015.

[50] B. Shen, P. Wang, R. Polson, and R. Menon, "An integratednanophotonics polarization beamsplitter with $2.4 \times 2.4 \mu \mathrm{m}^{2}$ footprint," Nat. Photonics, vol. 9, pp. 378-382, 2015.

[51] X. Wen, K. Xu, and Q. Song, "Design of a barcode-like waveguide nanostructurefor efficient chip-fiber coupling," Photonics Res., vol. 4, pp. 209-213, 2016.

[52] L. Lu, D. Liu, F. Zhou, et al., "Inverse-designed single-step-etched colorless $3 \mathrm{~dB}$ couplers based on RIE-lag-insensitive PhC-like subwavelength structures," Opt. Lett., vol. 41, pp. 5051-5054, 2016.

[53] B. Shen, R. Polson, and R. Menon, “Increasing the density of passive photonic-integrated circuits via nanophotonic cloaking," Nat. Commun., vol. 7, p. 13126, 2016.

[54] H. Jia, T. Zhou, X. Fu, J. Ding, and L. Yang, "Inverse-design and demonstration of ultracompact silicon meta-structure mode exchange device," ACS Photonics, vol. 5, pp. 1833-1838, 2018.

[55] Z. Yu, Y. Ma, and X. Sun, "Photonic welding points for arbitrary on-chip optical interconnects," Nanophotonics, vol. 7, pp. 1679-1686, 2018.

[56] Z. Yu, A. Feng, X. Xi, and X. Sun, "Inverse-designed low-loss and wideband polarization-insensitive silicon waveguide crossing," Opt. Lett., vol. 44, pp. 77-80, 2019.

[57] J. Kennedy and R. Eberhart, "Particle swarm optimization," in International Conference on Networks, vol. 4, Perth, WA, Australia, IEEE, 2002, pp. 1942-1948.

[58] Y. Zhang, S. Yang, T. Baehrjones, et al., "Compact and low loss Y-junction for submicron silicon waveguide," Opt. Express, vol. 21, pp. 1310-1316, 2013.

[59] Y. Shi and R. Eberhart, "A modified particle swarm optimizer," in IEEE International Conference on Evolutionary Computation, Anchorage, AK, USA, IEEE, 1998, pp. 69-73.

[60] S. Kirkpatrick, C. D. Gelatt, and M. P. Vecchi, "Optimization by simulated annealing," Science, vol. 220, pp. 671-680, 1983.

[61] J. Kim and J. D. O’Brien, "Optimization of a two-dimensional photonic-crystal waveguide branch by simulated annealing and the finite-element method," J. Opt. Soc. Am. B, vol. 21, pp. 289-295, 2004.

[62] K. Aydin, V. E. Ferry, R. M. Briggs, and H. A. Atwater, "Broadband polarization-independent resonant light absorption using ultrathin plasmonic super absorbers," Nat. Commun., vol. 2, p. 517, 2011. 
[63] J. Z. Samad, I. Sandeep, and M. Hossein, "Adaptive genetic algorithm for optical metasurfaces design," Sci. Rep., vol. 8, p. 11040, 2018.

[64] H. Liang, Q. Lin, X. Xie, et al., "Ultrahigh numerical aperture metalens at visible wavelengths," Nano Lett., vol. 18, pp. 4460-4466, 2018.

[65] Y. Cao, S. Li, L. R. Petzold, and R. Serban, “Adjoint sensitivity analysis for differential-algebraic equations: the adjoint DAE system and its numerical solution," SIAM J. Sci. Comput., vol. 24, pp. 1076-1089, 2002.

[66] A. L. Cauchy, "Methode generale pour la resolution des systemes d'equations simultanees," Comput. Rend. Sci., vol. 25, pp. 536-538, 1847.

[67] J. C. Meza, Steepest Descent, vol. 2, New York, USA, John Wiley \& Sons Inc., 2010, pp. 719-722.

[68] C. M. Lalaukeraly, S. Bhargava, O. D. Miller, and E. Yablonovitch, "Adjoint shape optimization applied to electromagnetic design,” Opt. Express, vol. 21, pp. 21693-21701, 2013.

[69] A. Michaels and E. Yablonovitch, "Leveraging continuous material averaging for inverse electromagnetic design," Opt. Express, vol. 26, pp. 31717-31737, 2018.

[70] N. Lebbe, A. Gliere, and K. Hassan, "High-efficiency and broadband photonic polarization rotator based on multilevel shape optimization,” Opt. Lett., vol. 44, pp. 1960-1963, 2019.

[71] M. P. Bendsøe and N. Kikuchi, "Generating optimal topologies in structural design using a homogenization method," Comput. Methods Appl. Mech. Eng., vol. 71, pp. 197-224, 1988.

[72] M. P. Bendsoe and O. Sigmund, Topology Optimization: Theory, Methods, and Applications, Berlin Heidelberg, NY, SpringerVerlag, 2003.

[73] J. Lu and J. Vuckovic, "Objective-first design of high-efficiency, small-footprint couplers between arbitrary nanophotonic waveguide modes," Opt. Express, vol. 20, pp. 7221-7236, 2012.

[74] Y. Liu, W. Sun, H. Xie, et al., "Adiabatic and ultracompact waveguide tapers based on digital metamaterials," IEEE J. Sel. Top. Quant. Electron., vol. 25, pp. 1-6, 2019.

[75] Y. Elesin, B. S. Lazarov, J. S. Jensen, and O. Sigmund, “Design of robust and efficient photonic switches using topology optimization," Photonics Nanostruct, vol. 10, pp. 153-165, 2012.

[76] M. Zhou, B. S. Lazarov, F. Wang, and O. Sigmund, "Minimum length scale in topology optimization by geometric constraints," Comput. Methods Appl. Mech. Eng., vol. 293, pp. 266-282, 2015.

[77] J. S. Jensen and O. Sigmund, "Topology optimization for nanophotonics," Laser Photonics Rev., vol. 5, pp. 308-321, 2011.

[78] P. I. Borel, A. Harpoth, L. H. Frandsen, and M. Kristensen, "Topology optimization and fabrication of photonic crystal structures," Opt. Express, vol. 12, pp. 1996-2001, 2004.

[79] L. H. Frandsen, A. Harpoth, P. I. Borel, and M. Kristensen, "Broadband photonic crystal waveguide $60^{\circ}$ bend obtained utilizing topology optimization," Opt. Express, vol. 12, pp. 5916-5921, 2004.

[80] P. I. Borel, B. Bilenberg, L. H. Frandsen, et al., "Imprinted siliconbased nanophotonics,"Opt. Express, vol. 15, pp. 1261-1266, 2007.

[81] L. H. Frandsen, Y. Elesin, and L. F. Frellsen, "Topology optimized mode conversion in a photonic crystal waveguide fabricated in silicon-on-insulator material," Opt. Express, vol. 22, pp. 8525-8532, 2014.

[82] L. F. Frellsen, Y. Ding, O. Sigmund, and L. H. Frandsen, “Topology optimized mode multiplexing in silicon-on-insulator photonic wire waveguides," Opt. Express, vol. 24, p. 16866, 2016.
[83] https://support.lumerical.com/hc/en-us/articles/ 360043165294.

[84] https://meep.readthedocs.io/en/latest/Python_Tutorials/ AdjointSolver/.

[85] S. Boyd, N. Parikh, E. Chu, B. Peleato, and J. Eckstein, "Distributed optimization and statistical learning via the alternating direction method of multipliers," Found Trends Mach. Learn., vol. 3, pp. 1-122, 2010.

[86] D. Vercruysse, N. V. Sapra, L. Su, R. Trivedi, and J. Vuckovic, "Analytical level set fabrication constraints for inverse design," Sci. Rep., vol. 9, p. 8999, 2019.

[87] L. Su, A. Y. Piggott, N. V. Sapra, J. Petykiewicz, and J. Vuckovic, "Inverse design and Demonstration of a compact on-chip narrowband three-channel wavelength demultiplexer," ACS Photonics, vol. 5, pp. 301-305, 2018.

[88] Y. A. Yilmaz, A. M. Alpkili, A. Yeltik, and H. Kurt, "Inverse design of efficient and compact $1 \times \mathrm{N}$ wavelength demultiplexer," Opt. Commun., vol. 454, p. 124522, 2019.

[89] J. Han, J. Huang, J. Wu, and J. Yang, "Inverse designed tunable four-channel wavelength demultiplexer," Opt. Commun., vol. 465, p. 125606, 2020.

[90] K. Y. Yang, J. Skarda, M. Cotrufo, et al., "Inverse-designed nonreciprocal pulse router for chip-based LiDAR," Nat. Photonics, vol. 14, pp. 369-374, 2020.

[91] C. Meng, J. Qiu, Y. Tian, Z. Ye, and J. Wu, "A broadband compact $1 \times 3$ power splitter designed with inverse design method," in International Conference on Optical Communications and Networks, Hangzhou, China, IEEE, 2016, pp. 1-3.

[92] F. Callewaert, S. Butun, Z. Li, and K. Aydin, "Inverse design of an ultra-compact broadband optical diode based on asymmetric spatial mode conversion," Sci. Rep., vol. 6, p. 32577, 2016.

[93] J. Huang, J. Yang, D. Chen, et al., "Ultra-compact broadband polarization beam splitter with strong expansibility," Photonics Res., vol. 6, pp. 574-578, 2018.

[94] L. Deng and D. Yu, "Deep learning: methods and applications," Found Trends Signal Process., vol. 7, pp. 3-4, 2014.

[95] Y. Lecun, Y. Bengio, and G. Hinton, “Deep learning," Nature, vol. 521, p. 436, 2015.

[96] D. E. Rumelhart, G. E. Hinton, and R. J. Williams, "Learning representations by back-propagating errors," Nature, vol. 323, pp. 533-536, 1986.

[97] A. Krizhevsky, I. Sutskever, and G. E. Hinton, "ImageNet classification with deep convolutional neural networks," Commun. ACM, vol. 60, pp. 84-90, 2017.

[98] R. Girshick, “Fast R-CNN," in International Conference on Computer Vision, Santiago Chile, IEEE, 2015, pp. 1440-1448.

[99] J. Redmon, S. K. Divvala, R. Girshick, and A. Farhadi, "You only look once: unified, real-time object betection," in IEEE Conference on Computer Vision and Pattern Recognition, pp. 779-788, 2016. https://doi.org/10.1109/CVPR.2016.91.

[100] J. Devlin, M. Chang, K. Lee, and K. Toutanova, "BERT: Pretraining of deep bidirectional transformers for language understanding," arXiv preprint, pp. arXiv:1810.04805v2, 2018.

[101] G. Hinton, L. Deng, D. Yu, et al., "Deep neural networks for acoustic modeling in speech recognition: the Shared Views of Four Research Groups," IEEE Signal Process. Mag., vol. 29, pp. 82-97, 2012.

[102] D. Amodei, S. Ananthanarayanan, R. Anubhai, et al., "Deep speech 2: end-to-end speech recognition in English and 
Mandarin," in International Conference on Machine Learning, NY, USA, JMLR, 2016, pp. 173-182.

[103] D. Silver, A. Huang, C. J. Maddison, et al., "Mastering the game of go with deep neural networks and tree search," Nature, vol. 529, pp. 484-489, 2016.

[104] H. Kabir, Y. Wang, M. Yu, and Q. Zhang, "Neural network inverse modeling and applications to microwave filter design," IEEE Trans. Microw. Theor. Tech., vol. 56, pp. 867-879, 2008.

[105] G. Carleo and M. Troyer, "Solving the quantum many-body problem with artificial neural networks," Science, vol. 355, pp. 602-606, 2017.

[106] F. A. Faber, L. Hutchison, B. Huang, et al., “Machine learning prediction errors better than DFT accuracy," arXiv preprint, pp. arXiv:1702.05532v2, 2017. https://doi.org/10.1021/acs.jctc. $7 \mathrm{~b} 00577$.

[107] S. So, T. Badloe, J. Noh, J. Rho, and J. Bravoabad, “Deep learning enabled inverse design in nanophotonics," Nanophotonics, vol. 9, pp. 1041-1057, 2020.

[108] J. Jiang, M. Chen, and J. A. Fan, "Deep neural networks for the evaluation and design of photonic devices," arXiv preprint, pp. arXiv:2007.00084v1, 2020.

[109] D. Liu, Y. Tan, E. Khoram, and Z. Yu, "Training deep neural networks for the inverse design of nanophotonic structures," ACS Photonics, vol. 5, pp. 1365-1369, 2018.

[110] R. B. Marimont and M. B. Shapiro, "Nearest neighbour searches and the curse of dimensionality," IMA J. Appl. Math., vol. 24, pp. 59-70, 1979.

[111] A. Zimek, E. Schubert, and H-P. Kriegel, "A survey on unsupervised outlier detection in high-dimensional numerical data," Stat. Anal. Data Min., vol. 5, pp. 363-387, 2012.

[112] P. John, S. Yichen, J. Li, et al., "Nanophotonic particle simulation and inverse design using artificial neural networks," Sci. Adv., vol. 4, p. eaar4206, 2018.

[113] M. Itzik, M. Michael, N. Achiya, A. Uri, W. Lior, and S. Haim, "Plasmonic nanostructure design and characterization via deep learning," Light Sci. Appl., vol. 7, p. 60, 2018.

[114] Z. Liu, D. Zhu, S. P. Rodrigues, K. T. Lee, and W. Cai, “Generative model for the inverse design of metasurfaces," Nano Lett., vol. 18, pp. 6570-6576, 2018.

[115] I. Sajedian, J. Kim, and J. Rho, "Finding the optical properties of plasmonic structures by image processing using a combination of convolutional neural networks and recurrent neural networks," Microsyst. Nanoeng., vol. 5, pp. 1-8, 2019.

[116] W. Ma, F. Cheng, and Y. Liu, "Deep-learning-enabled ondemand design of chiral metamaterials," ACS Nano, vol. 12, pp. 6326-6334, 2018.

[117] T. Zhang, J. Wang, Q. Liu, et al., "Efficient spectrum prediction and inverse design for plasmonic waveguide systems based on artificial neural networks," Photonics Res., vol. 7, pp. 368-380, 2019.

[118] L. Pilozzi, F. A. Farrelly, G. Marcucci, and C. Conti, "Machine learning inverse problem for topological photonics," Commun. Phys., vol. 1, p. 57, 2018.

[119] J. Jiang, D. Sell, S. Hoyer, J. Hickey, J. Yang, and J. A. Fan, “Freeform diffractive metagrating design based on generative adversarial networks," ACS Nano, vol. 13, pp. 8872-8878, 2019.

[120] W. Ma, F. Cheng, Y. Xu, Q. Wen, and Y. Liu, "Probabilistic representation and inverse design of metamaterials based on a deep generative model with semi-supervised learning strategy," Adv. Mater., vol. 31, p. 1901111, 2019.
[121] D. Melati, Y. Grinberg, M. K. Dezfouli, et al., “Mapping the global design space of nanophotonic components using machine learning pattern recognition," Nat. Commun., vol. 10, pp.1-9, 2019.

[122] Z. Liu, Z. Zhu, and W. Cai, "Topological encoding method for data-driven photonics inverse design," Opt. Express, vol. 28, pp. 4825-4835, 2020.

[123] Y. Kiarashinejad, S. Abdollahramezani, and A. Adibi, “Deep learning approach based on dimensionality reduction for designing electromagnetic nanostructures," NPJ Comput. Mater., vol. 6, pp. 1-12, 2019.

[124] M. H. Tahersima, K. Kojima, T. Koikeakino, et al., "Deep neural network inverse design of integrated photonic power splitters," Sci. Rep., vol. 9, p. 1368, 2019.

[125] C. Nadell, B. Huang, J. Malof, and W. Padilla, "Deep learning for accelerated all-dielectric metasurface design," Opt. Express, vol. 27, pp. 27523-27535, 2019.

[126] M. TaherSima, K. Kojima, T. Koike-Akino, et al., "Nanostructured photonic power splitter design via convolutional neural networks," in Conference on Lasers and Electro-Optics, San Jose, CA, USA, IEEE, 2019, https://doi.org/ 10.1364/CLEO_SI.2019.SW4J.6.

[127] P. Sanchis, P. Villalba, F. Cuesta, et al., "Highly efficient crossing structure for silicon-on-insulator waveguides," Opt. Lett., vol. 34, pp. 2760-2762, 2009.

[128] Y. Zhang, S. Yang, A. E. Lim, et al., "A CMOS-compatible, low-loss, and low-crosstalk silicon waveguide crossing," IEEE Photonics Technol. Lett., vol. 25, pp. 422-425, 2013.

[129] C. Koos, C. G. Poulton, L. Zimmermann, L. Jacome, J. Leuthold, and W. Freude, "Ideal bend contour trajectories for single-mode operation of low-loss overmoded waveguides," IEEE Photonics Technol. Lett., vol. 19, pp. 819-821, 2007.

[130] F. Zhou, L. Lu, M. Zhang, et al., "Ultra-compact, low-loss and low-crosstalk wavelength demultiplexer for CWDM system based on the photonic-crystal-like metamaterial structure," in Conference on Lasers \& Electro-optics, San Jose, CA, USA, IEEE, 2017, https://doi.org/10.1364/cleo_at.2017.jth2a.101.

[131] W. Chang, L. Lu, X. Ren, et al., "Ultracompact dual-mode waveguide crossing based on subwavelength multimodeinterference couplers," Photonics Res., vol. 6, pp. 660-665, 2018.

[132] W. Bogaerts and S. K. Selvaraja, "Compact single-mode silicon hybrid rib/strip waveguide with adiabatic bends," IEEE Photonics J., vol. 3, pp. 422-432, 2011.

[133] Y. Liu, W. Sun, H. Xie, et al., "Very sharp adiabatic bends based on an inverse design," Opt. Lett., vol. 43, pp. 2482-2485, 2018.

[134] C. Sun, Y. Yu, G. Chen, and X. Zhang, "Ultra-compact bent multimode silicon waveguide with ultralow inter-mode crosstalk," Opt. Lett., vol. 42, pp. 3004-3007, 2017.

[135] Q. Xu, B. Schmidt, J. Shakya, and M. Lipson, "Cascaded silicon micro-ring modulators for WDM optical interconnection," Opt. Express, vol. 14, pp. 9431-9436, 2006.

[136] Q. Fang, T. Liow, J. Song, et al., "WDM multi-channel silicon photonic receiver with 320 Gbps data transmission capability," Opt. Express, vol. 18, pp. 5106-5113, 2010.

[137] F. Xia, M. Rooks, L. Sekaric, and Y. Vlasov, “Ultra-compact high order ring resonator filters using submicron silicon photonic wires for on-chip optical interconnects," Opt. Express, vol. 15, pp. 11934-11941, 2007. 
[138] S. Inao, T. Sato, S. Sentsui, T. Kuroha, and Y. Nishimura, "Multicore Optical Fibe," in Optical Fiber Communication Conference, Washington, DC, USA, WB1, 1979.

[139] D. J. Richardson, J. M. Fini, and L. E. Nelson, "Space-division multiplexing in optical fibres," Nat. Photonics, vol. 7, pp. 354-362, 2013.

[140] L. Luo, N. Ophir, C. P. Chen, et al., "WDM-compatible modedivision multiplexing on a silicon chip," Nat. Commun., vol. 5, p. 3069, 2014.

[141] W. Chang and M. Zhang, "Silicon-based multimode waveguide crossings," J. Phys. Photonics, vol. 2, 2020, https://doi.org/10. 1088/2515-7647/ab8698.

[142] W. Chang, L. Lu, X. Ren, et al., “Ultra-compact mode (de) multiplexer based on subwavelength asymmetric Y-junction," Opt. Express, vol. 26, pp. 8162-8170, 2018.

[143] H. Xie, Y. Liu, S. Wang, et al., "Highly compact and efficient fourmode multiplexer based on pixelated waveguides," IEEE Photonics Technol. Lett., vol. 32, pp. 166-169, 2020.

[144] L. Lu, M. Zhang, F. Zhou, and D. Liu, "An ultra-compact colorless 50:50 coupler based on phc-like metamaterial structure," in Optical Fiber Communication Conference, Anaheim, CA, USA, IEEE, 2016, pp. 1-3.

[145] Y. Tang, K. Kojima, T. Koikeakino, et al., "Generative deep learning model for a multi-level nano-optic broadband power splitter," in Optical Fiber Communication Conference, San Diego, CA, USA, IEEE, 2020, p. Th1A.1.

[146] K. Xu, L. Liu, X. Wen, et al., "Integrated photonic power divider with arbitrary power ratios," Opt. Lett., vol. 42, pp. 855-858, 2017.

[147] H. Xie, Y. Liu, W. Sun, et al., "Inversely designed 1×4 power splitter with arbitrary ratios at $2-\mu \mathrm{m}$ spectral band," IEEE Photonics J., vol. 10, pp. 1-6, 2018.

[148] H. Ma, J. Huang, K. Zhang, and J. Yang, “Arbitrary-direction, multichannel and ultra-compact power splitters by inverse design method," Opt. Commun., vol. 462, p. 125329, 2020

[149] W. Chang, L. Lu, D. Liu, and M. Zhang, "An ultra-compact colorless dual-mode $3 \mathrm{~dB}$ power splitter based on axisymmetrical subwavelength structure," in Conference on Lasers and Electro Optics, vol. 47, 2018, p. JW2A.

[150] H. Xie, Y. Liu, Y. Wang, et al., "An ultra-compact 3-dB power splitter for three modes based on pixelated meta-structure," IEEE Photonics Technol. Lett., vol. 32, pp. 341-344, 2020.

[151] L. H. Frandsen and O. Sigmund, "Inverse design engineering of all-silicon polarization beam splitters," in Proc. SPIE, vol. 9756, San Francisco, California, USA, SPIE, 2016, p. 97560Y. Available at: http://proceedings.spiedigitallibrary.org/ on 04/06/2016.

[152] A. Y. Piggott, J. Lu, T. M. Babinec, K. G. Lagoudakis, J. Petykiewicz, and J. Vuckovic, "Inverse design and implementation of a wavelength demultiplexing grating coupler," Sci. Rep., vol. 4, p. 7210, 2015.

[153] K. O. Abrokwah, Characterization and Modeling of Plasma Etch Pattern Dependencies in Integrated Circuits, Cambridge, MA, USA, Massachusetts Institute of Technology, 2006, pp. 106-107.

[154] B. Bourdin, "Filters in topology optimization," Int. J. Numer. Methods Eng., vol. 50, pp. 2143-2158, 2001.

[155] 0. Sigmund, "Morphology-based black and white filters for topology optimization," Struct. Multidiscip. Optim., vol. 33, pp. 401-424, 2007.
[156] E. Khoram, X. Qian, M. Yuan, and Z. Yu, "Controlling the minimal feature sizes in adjoint optimization of nanophotonic devices using b-spline surfaces," Opt. Express, vol. 28, pp. 7060-7069, 2020.

[157] F. Wang, B. S. Lazarov, and O. Sigmund, “On projection methods, convergence and robust formulations in topology optimization," Struct. Multidiscip. Optim., vol. 43, pp. 767-784, 2011.

[158] R. Bruck, K. Vynck, P. Lalanne, et al., "All-optical spatial light modulator for reconfigurable silicon photonic circuits," Optica, vol. 3, pp. 396-402, 2016

[159] J. Wang, Y. Shi, T. Hughes, Z. Zhao, and S. Fan, "Adjoint-based optimization of active nanophotonic devices," Opt. Express, vol. 26, pp. 3236-3248, 2018.

[160] D. Vercruysse, N. V. Sapra, L. Su, and J. Vuckovic, "Dispersion engineering with photonic inverse design," IEEE J. Sel. Top. Quant. Electron., vol. 26, pp. 1-6, 2020.

[161] R. Pestourie, C. Perezarancibia, Z. Lin, W. Shin, F. Capasso, and S. G. Johnson, "Inverse design of large-area metasurfaces," Opt. Express, vol. 26, pp. 33732-33747, 2018.

[162] A. S. Backer, "Computational inverse design for cascaded systems of metasurface optics," Opt. Express, vol. 27, pp. 30308-30331, 2019.

[163] E. W. Wang, D. Sell, T. Phan, and J. A. Fan, "Robust design of topology-optimized metasurfaces," Opt. Mater. Express, vol. 9, pp. 469-482, 2019

[164] J. A. Bossard, L. Lin, S. Yun, L. Liu, D. H. Werner, and T. S. Mayer, "Near-Ideal optical metamaterial absorbers with super-octave bandwidth," ACS Nano, vol. 8, pp. 1517-1524, 2014.

[165] H. Chung and O. D. Miller, "High-NA achromatic metalenses by inverse design," Opt. Express, vol. 28, pp. 6945-6965, 2020.

[166] J. Li, L. Bao, S. Jiang, et al., "Inverse design of multifunctional plasmonic metamaterial absorbers for infrared polarimetric imaging," Opt. Express, vol. 27, pp. 8375-8386, 2019.

[167] Y. Chen, J. Zhu, Y. Xie, N. Feng, and Q. Liu, "Smart inverse design of graphene-based photonic metamaterials by an adaptive artificial neural network," Nanoscale, vol. 11, pp. 9749-9755, 2019.

[168] A. D. Phan, C. V. Nguyen, P. T. Linh, et al., "Deep learning for the inverse design of mid-infrared graphene plasmons," Crystals, vol. 10, p. 125, 2020

[169] C. Dory, D. Vercruysse, K. Y. Yang, et al., "Inverse-designed diamond photonics," Nat. Commun., vol. 10, pp. 1-7, 2019.

[170] S. S. Nanthakumar, X. Zhuang, H. Park, C. Nguyen, Y. Chen, and T. Rabczuk, "Inverse design of quantum spin hall-based phononic topological insulators," J. Mech. Phys. Solid., vol.125, pp. 550-571, 2019.

[171] Z. Xie, T. Lei, H. Qiu, Z. Zhang, H. Wang, and X. Yuan, "Broadband on-chip photonic spin Hall element via inverse design," Photonics Res., vol. 8, pp. 121-126, 2020.

[172] G. Angeris, J. Vuckovic, and S. P. Boyd, "Computational bounds for photonic design,” ACS Photonics, vol. 6, pp. 1232-1239, 2019.

[173] L. Su, D. Vercruysse, J. Skarda, N. V. Sapra, J. A. Petykiewicz, and J. Vuckovic, "Nanophotonic inverse design with SPINS: software architecture and practical considerations," Appl. Phys. Rev., vol. 7, 2020, p. 011407. 